Pacific Journal of Mathematics

HOLONOMY GROUPS OF INDEFINITE METRICS 


\title{
HOLONOMY GROUPS OF INDEFINITE METRICS
}

\author{
H. WU
}

This paper studies the holonomy group of a riemannian manifold whose metric is allowed to have arbitrary signature; it is meant to supplement the works of Borel, Lichnerowicz and Berger on riemannian manifolds with positive definite metric. We first show that each such holonomy group can be decomposed into the direct product of a finite number of weakly irreducible subgroups of the pseudo-orthogonal group. Those weakly irreducible subgroups which are not irreducible (in the usual sense) we call $S-W$ irreducible. So our investigation is reduced to that of these $S-W$ irreducible holonomy groups. We actually construct a large class of symmetric spaces with $S-W$ irreducible holonomy groups and for the nonsymmetric case, we give an indication of their abundant existence. On the other hand, not every $S-W$ irreducible group can be realized as a holonomy group; this fact is shown by an explicit example. We then study the closedness question of $S-W$ irreducible subgroups in general, and of holonomy groups in particular. It turns out that algebraic holonomy groups (and hence $S-W$ irreducible subgroups in general) need not be closed in $G l_{n}$ but that holonomy groups of symmetric riemannian manifolds of any signature are necessarily closed. Sufficient conditions are also given in order that an $S-W$ irreducible subgroup be closed. Finally, we produce various counterexamples to show that many facts known to hold in the positive definite case fail when the metric is allowed to be indefinite.

In two exhaustive works [2], [3], Berger has given a complete classification of possible candidates for irreducible holonomy groups of riemannian manifolds. (In this paper, "holonomy groups" is synonymous with "the identity component of the homogeneous holonomy group" and riemannian manifolds carry metrics of arbitrary signatures. For all relevant conventions and definitions, see Section 2). Since Borel and Lichnerowicz have shown [4] that for the positive definite case, every holonomy group is the direct product of irreducible subgroups of the orthogonal group, the consideration of irreducible holonomy groups alone is sufficient for that case. On the other hand, if the metric is indefinite, the situation becomes different. Defining a subgroup of the pseudo-orthogonal group $P O(V)$ to be weakly irreducible if and only if it leaves invariant only proper degenerate subspaces of $V$, we have the following simple but basic result.

THEOREM 1. The holonomy group of a riemannian manifold is 
the direct product of a finite numbsr of its normal subgroups which are all weakly irreducible. This direct product decomposition is unique (up to order) if the holonomy group possesses a nondegenerate maximal trivial subspace (see Section 2 for definition).

(This theorem is closely related to those stated in Appendices I and II at the end of the paper.)

The main concern of this paper will be this class of weakly irreducible subgroups. The sub-class of irreducible subgroups is already covered by Berger's papers so that we wish to exclude it from our consideration from now on. We call a weakly irreducible group strictly weakly irreducible if and only if it leaves invariant a proper (degenerate) subspace. The first natural question to ask is whether $S-W$ irreducible (abbreviation for strictly weakly irreducible) holonomy groups exist at all. It is easy to see that the holonomy group of a two-dimensional Lorentz manifold is necessarily $S-W$ irreducible because the isotropic directions are preserved. But dimension 2 has a habit of being the exceptional case in the general theory, and one would like to have less trivial examples. The following theorem gives an abundant supply of them. First we describe a general procedure of sending an arbitrary Lie algebra $\mathfrak{g}$ to a symmetric riemannian manifold $M(\mathfrak{g})$. Let $\mathrm{g}^{*}$ be the vector space dual of $\mathfrak{g}$ considered as an abelian Lie algebra, and let $\mathfrak{g}^{\prime}$ be the semi-direct product of $g$ and $g^{*}$ with respect to the dual of the adjoint representation of $g$. Then the natural pairing between $g$ and $\mathrm{g}^{*}$ induces an inner product on $\mathrm{g}^{\prime}$ which has the same number of positive and negative squares and which turns out to be ad-invariant. Once we have this $g^{\prime}$ equipped with an ad-invariant inner product, we can imitate the case of compact Lie algebras to construct a symmetric space $M(\mathfrak{g})$, diffeomorphic to the simply connected group corresponding to $g^{\prime}$. (Cf. [8] for details). With a minor refinement of this technique and restricting oneself to solvable $\mathfrak{g}$, one has the fairly precise:

THEOREM 2. To each solvable Lie algebra $g$ of dimension d can be associated a riemannian manifold of dimension $2 d$ such that $M=M_{0} \times M_{1} \times \cdots \times M_{k}$ (isometry) where $M_{0}$ is isometric to an inner-product vector space, and each $M_{i}$ for $i>0$ is a $S$-W irreducible symmetric space diffeomorphic to a euclidean space. If $[\mathfrak{g}, \mathscr{D g}] \neq 0$, then $k>0$. If furthermore $\left[\mathrm{g}, \mathscr{D}^{2} \mathrm{~g}\right] \neq 0$, then the holonomy group of at least one of the $M_{i}^{\prime}$ s is solvable but not abelian.

In particular, when $g$ is the matrix algebra of super-triangular matrices with trace equal to zoro, then this $M$ is itself $S-W$ irreducible, i.e. $M_{0}=\{0\}$, and $k=1$.

The explicit construction of $M$ from a given $g$ can be quite elabo- 
rate, and it is in general rather tedious to compute the holonomy groups of the $M_{i}^{\prime} s$. For this and other reasons to be made clear presently, we now consider two concrete examples which are not constructed by this procedure.

Example 3. (a) $R^{3}$ can be made into a $S-W$ irreducible riemannian symmetric space whose holonomy group is the following one-parameter subgroup of $S O(2,1)$ : relative to an orthonormal basis $\left\{e_{1}, e_{2}, e_{3}\right\}$ of type $(+,+,-)$,

$$
H=\left[\begin{array}{ccc}
1-t^{2} & -t & t^{2} \\
t & 1 & -t^{2} \\
-t^{2} & t & 1+t^{2}
\end{array}\right] \quad t \in \boldsymbol{R}
$$

(b) There exists a $S-W$ irreducible kahlerian symmetric space diffeomorphic to $\boldsymbol{R}^{4}$ whose holonomy group is the following one-parameter subgroup of $S O(2,2)$ : relative to an orthonormal basis $\left\{e_{1}, e_{2}, e_{3}, e_{4}\right\}$ of type $(+,+,-,-)$,

$$
H=\left[\begin{array}{cccc}
1 & -t & 0 & t \\
t & 1 & -t & 0 \\
0 & -t & 1 & t \\
t & 0 & -t & 1
\end{array}\right] \quad t \in \boldsymbol{R}
$$

Our interest in these spaces lies in the fact that they serve as a good indication of how pathological $S-W$ irreducible manifolds can be, i.e., pathological in the light of corresponding situations of irreducible manifolds. One knows that the full isometry group of an irreducible (positive definite) riemannian symmetric space is semisimple and coincides with its full group of affine diffeomorphisms. It will be shown in Section 7, however, that the full isometry group of the space in Example 3 (a) is solvable and strictly smaller than the group of all affine diffeomorphisms; also the algebra of the full isometry group of the space of Example $3(\mathrm{~b})$ has a nontrivial Levi decomposition. Example 3 (b) is of interest in other connections as well: we shall show with its help that the condition for uniqueness of decomposition in Theorem 1 cannot be relaxed, (thus answering at the same time the question raised at the end of [15]). Note first that this holonomy group has an isotropic maximal trivial subspace span $\left\{e_{1}+e_{3}, e_{2}+e_{4}\right\}$, i.e., it acts trivially on this subspace and on no bigger one. Consider $M=\boldsymbol{R}^{4} \times \boldsymbol{R}^{4}$, where each factor is equipped with the riemannian structure of Theorem 3 (b). Choose an orthonormal basis $\left\{e_{1}, \cdots, e_{4}\right\}$ (resp. $\left\{f_{1}, \cdots, f_{4}\right\}$ ) of $\left(\boldsymbol{R}_{0}^{4}, 0\right)$ (resp. $\left(0, \boldsymbol{R}_{0}^{4}\right)$ ), where 0 denote the origin, of the type appearing in Theorem $3(\mathrm{~b})$. Then corresponding to this decomposition 
of $M_{(0,0)}=\boldsymbol{R}_{0}^{4} \oplus \boldsymbol{R}_{0}^{4}$, we get the usual decomposition of the holonomy group of $M$ at $(0,0)$, namely, $H=H_{1} \times H_{2}$, where $H_{1}$ (resp. $\left.H_{2}\right)$ is the holonomy group of the first (resp. second) factor. Now let

$$
\begin{aligned}
& W_{1}=\operatorname{span}\left\{e_{1}-\left(f_{1}+f_{3}\right), e_{2}, e_{3}+\left(f_{1}+f_{3}\right), e_{4}\right\}, \\
& W_{2}=\operatorname{span}\left\{f_{1}+\left(e_{1}+e_{3}\right), f_{2}, f_{3}-\left(e_{1}+e_{3}\right), f_{4}\right\} .
\end{aligned}
$$

Then $M_{(0,0)}=W_{1} \oplus W_{2}$ is another decomposition of $M_{(0,0)}$ into mutually orthogonal nondegenerate subspaces which are each preserved by the holonomy group. There is, consequently, a corresponding decomposition of the holonomy group (cf. Appendix I) $H=H_{1}^{\prime} \times H_{2}^{\prime}$, such that $H_{1}^{\prime}$ (resp. $H_{2}^{\prime}$ ) acts weakly irreducibly on $W_{1}$ (resp. $W_{2}$ ) and acts trivially on $W_{2}$ (resp. $\left.W_{1}\right)$. Here are, then, two distinct decompositions of the holonomy group as a direct product of its normal subgroups. Next, we shall show-still using Example 3 (b) - in a very striking way the necessity of the nondegeneracy assumption in the de Rham Decomposition Theorem, ([15] especially Section 5, (3)). Returning to the notation of Theorem $3(\mathrm{~b})$, let $U_{1}=\operatorname{span}\left\{\alpha e_{1}+\beta e_{3}, e_{2}+e_{4}\right\}, U_{2}=\operatorname{span}\left\{e_{1}+e_{3}\right.$, $\left.\gamma e_{3}+\delta e_{4}\right\}, \alpha, \beta, \gamma, \delta \in \boldsymbol{R}$. Now if $\alpha \neq \beta, \gamma \neq \delta$, it is easy to see that $\boldsymbol{R}_{0}^{4}=U_{1} \oplus U_{2}$ and that both $U_{1}, U_{2}$ are left invariant by the holonomy group. (If we let $\alpha=-\beta, \gamma=-\delta, U_{1}$ and $U_{2}$ are even both isotropic.) But we know that $\boldsymbol{R}^{4}$ in this riemannian structure cannot even be affinely diffeomorphic to a direct product because of the weak irreducibility of its holonomy group (Main Theorem of [16]). So, we have an example of a manifold such that its holonomy group leaves invariant an infinite number of pairs of supplementary subspaces, but it is not affinely diffeomorphic to a direct product.

Theorem 2 and Example 3 suggest that there are probably too many indefinite riemannian symmetric spaces to be classified completely. For the same reason, it is unrealistic to expect to be able to enumerate all $S-W$ irreducible subgroups of $P O(V)$ as possible candidates for holonomy groups. One suspects, however, that not every $S-W$ irreducible group can be a holonomy group, and the following confirms this.

EXAMPLE 4. There are both closed and nonclosed subgroups of $S O(5,1)$ which are $S-W$ irreducible but can never be the holonomy groups of riemannian manifolds.

This example shows, incidentally, that $S-W$ irreducible subgroups are not necessarily closed in the general linear group. Since BorelLichnerowicz [4] proved that all holonomy groups in the positive definite case are closed, one would like to know if this is still true in the indefinite case. This is a fairly delicate question and we handle it 
exclusively via the Lie algebra. Our solution to this problem is not complete, but we have probably exhausted all that this algebraic approach promises to give. The "positive" result in this direction is the following.

THEOREM 5. The following subgroups of the general linear group are closed:

(a) Reductive weakly irreducible subgroups.

(b) Weakly irreducible subgroups of $P O(V), \operatorname{dim} V<6$.

(c) Holonomy groups of affine symmetric spaces.

Part (c) of course includes as a special case the fact that holonomy groups of riemannian symmetric spaces are closed in the general linear group. It should be noted that even in the riemannian case, (c) is not a consequence of (a), as Theorem 2 shows. (In Section 5, a symmetric space with solvable but nonabelian holonomy group will actually be constructed explicity as a by-product of something else.) In view of the fact that the holonomy groups of symmetric spaces can have such varied properties algebraically, that it should nonetheless be closed seems quite surprising. Parts (a) and (b) are also optimal, as is clear from the other results given herein. Our answer to the general question of holonomy groups of nonsymmetric riemannian manifolds is a partial one:

EXAMPLE 6. There exists an algebraic kahlerian holonomy group (see Section 2 for definition) which is a nonclosed subgroup of $S O(4,2)$.

The question of when an algebraic holonomy group can be realized as an honest holonomy group of a manifold is a deep and unsolved problem, (unsolved even in the positive definite case). On the other hand, we have been able to produce numerous nonclosed algebraic holonomy groups in various dimensions (that of Theorem 6 being the simplest) and it seems to us unlikely that the holonomy group of a general riemannian manifold needs to be closed. Using Nijenhuis' Theorem [12] (or p. 153 of [10]), one can of course try to substantiate this statement, but the large amount of computations required for this task absolutely defeats us.

In the foregoing, only holonomy groups of symmetric spaces have been considered because they are the easiest to compute. It is also not difficult to construct nonsymmetric riemannian spaces in low dimensions with $S-W$ irreducible holonomy groups. We give the following theorem as a sample with a view to counteract the impression that $S-W$ irreducibility is a special property of symmetric spaces. 
EXAMPLE 7. There exists an analytic riemannian structure on $\boldsymbol{R}^{3}$ with the same holonomy group as that of Theorem 3 (a). This riemannian structure is NOT symmetric.

The paper is organized as follows. Section 2 summarizes all definitions and conventions employed throughout this paper. Sections 3-6 give the proofs of the above theorems. The last section, Section 7 , contains further comments of a general nature. In two appendices at the end of the paper are to be found some additions and minor corrections to [15] and [16].

Finally, I would like to express my deep sense of gratitude to Professor Armand Borel; his help proved invaluable in the final stage of preparation of this paper.

2. The following is a list of definitions and notational conventions. All manifolds and all geometric structures on them are $C^{\infty}$. All manifolds and Lie groups are assumed connected unless otherwise specified. An affine diffeomorphism between two affinely connected manifolds (i.e. manifolds carrying an affine connection) is a diffeomorphism that preserves the connections. We shall be interested only in globally symmetric spaces (affine, riemannian or hermitian) so that the adjective "globally" will be omitted everywhere. Inner products on vector spaces as well as metrics on manifolds can have arbitrary signatures. A basis $\left\{e_{1}, \cdots, e_{d}\right\}$ of an inner-product space is said to be orthonormal of type $(+, \cdots,+,-, \cdots,-)(p+' s$ and $(d-p)-' s)$ if and only if $\left\langle e_{i}, e_{j}\right)=\delta_{i j}$ for $1 \leqq i, j \leqq p,\left\langle e_{m}, e_{n}\right\rangle=-\delta_{m n}$ for $p+1 \leqq m, n \leqq d$, and $\left\langle e_{\alpha}, e_{\beta}\right\rangle=0$ if $\alpha \neq \beta$. A subspace of an inner-product space is said to be nondegenerate (resp. degenerate, isotropic) if and only if the restriction of the inner product to it is nondegenerate (resp. degenerate, identically zero.)

We denote Lie groups by capital Latin letters $G, H, K$ and their Lie algebras by the corresponding small German letters $\mathfrak{g}, \mathfrak{f}, \mathfrak{h}$. As usual, we denote the $n$-th derived algebra of $\mathfrak{g}$ by $\mathscr{D}^{n} \mathfrak{g}$, i.e. $\mathscr{D}^{\prime} \mathfrak{g}=$ $\mathscr{D} \mathfrak{g}=[\mathfrak{g}, \mathfrak{g}], \mathscr{D}^{n} \mathfrak{g}=\mathscr{D}\left(\mathscr{D}^{n+1} \mathfrak{g}\right) . \quad A d$ and $a d$ denote the adjoint representations of $G$ and $g$. We introduce an abbreviation in this connection. Suppose a Lie subgroup $K \subseteq G$ leaves a subspace $\mathfrak{p}$ of $\mathfrak{g}$ invariant via the $A d$ action, then we denote by $A d_{\mathfrak{p}}(K)$ the subgroup of $G(\mathfrak{p})$ which is the restriction of $A d(K)$ to $\mathfrak{p}$. Similarly for $a d$.

In general $H$ is the generic symbol of holonomy groups and $\mathfrak{h}$, of holonomy algebras. We denote (the identify component of) the full group of isometries of an inner product space $V$ by $P O(V)$ when we are not concerned with the signature, and by $S O(p, d-p)$ when we are. The corresponding Lie algebras are then $\mathfrak{p o}(V)$ and $\mathfrak{s} \mathfrak{0}(p, d-p)$. 
$P O(V)$ is a subgroup of the automorphism group $\operatorname{Gr}(V)$ of $V$ which is usually identified with the group of all nonsingular $d \times d$ matrices in the presence of a basis; $\mathfrak{p o}(V)$ is then a Lie subalgebra of the full matrix algebra $g l(V)$, which is identified via the same basis with $\operatorname{Hom}(V, V)$.

A subgroup $G$ of $P O(V)$ is said to act weakly irreducibly on $V$ if and only if the proper subspaces of $V$ left invariant by $G$ are all degenerate; if furthermore $G$ does leave invariant a proper (degenerate) subspace, $G$ is said to act strictly weakly irreducibly (abbrev. $=S-W$ irreducibly). We also refer to $G$ as a weakly irreducible group (resp. $S-W$ irreducible group). Note that if a Lie group acts on a vector space, so does its algebra in a natural way. Thus, all concepts introduced pertaining to the group can be transferred verbatim to the algebra. The maximal subspace on which $G$ acts trivially is called the maximal trivial subspaces of $G$ in $V$. Most of the time, we will deal with a holonomy group $H$; in that case $H$ will be understood to be acting on some tangent space of the manifold and references to the latter will generally be omitted. Also, we follow the standard practice of defining a manifold to be weakly (resp. $S-W$ ) irreducible if its holonomy group is weakly (resp. $S-W$ ) irreducible.

We now give a series of definitions centering around curvature tensors. If $F: U \wedge U \rightarrow W$ is a linear map between vector spaces, we denote the value of $F$ at $x \wedge y$ by $F_{x y}$.

DeFINITION 1. A curvature tensor on a vector space $V$ is a linear $\operatorname{map} R: V \wedge V \rightarrow \operatorname{Hom}(V, V)$ such that

$$
R_{x y} z+R_{y z} x+R_{z x} y=0, \quad \forall x, y, z \in V .
$$

Now suppose on $V$ is defined an inner product $\langle,>, R$ is called a riemannian curvature tensor if and only if it further satisfies

$$
\left\langle R_{x y} z, w\right\rangle=-\left\langle z, R_{x y} w\right\rangle, \quad \forall x, y, z, w \in V .
$$

(In other words, if and only if $R$ takes value in $\mathfrak{p o}(V)$.) If, in addition, $V$ possesses a complex structure compatible with its inner product, i.e., if there exists a $J: V \rightarrow V$ such that $J^{2} x=-x$, and $\langle J x, J y\rangle=\langle x, y\rangle$, $\forall x, y \in V$, then $R$ is said to be a kahlerian curvature tensor when it satisfies (1), (2), and

$$
R_{x y} \circ J=J \circ R_{x y}, \quad \forall x, y \in V .
$$

We recall that a riemannian curvature tensor automatically satisfies the identity:

$$
\left\langle R_{x y} z, w\right\rangle=\left\langle R_{z w} x, y\right\rangle, \quad \forall x, y, z, w \in V
$$


and that a kahlerian curvature tensor automatically satisfies the identity:

$$
R_{J x J y}=R_{x y} \quad \forall x, y \in V .
$$

This will be made use of below.

Definition 2. A connected Lie subgroup $H^{*}$ of $G \mathrm{r}(V)$ is called an algebraic holonomy group if and only if there exists curvature tensors $\left\{R^{1}, \cdots, R^{r}\right\}$ on $V$ such that the Lie algebra $\mathfrak{h}^{*}$ of $H^{*}$ is exactly the linear span of the $R_{x y}^{i}$, all $x, y \in V, i=1, \cdots, r$. An algebraic riemannian (resp. kahlerian) holonomy group is an algebraic holonomy group such that the $\left\{R^{1}, \cdots, R^{r}\right\}$ are all riemannian (resp. kahlerian) curvature tensors. Thus, if an algebraic holonomy group is either riemannian or kahlerian, it is a subgroup of $P O(V)$.

REMARK 1. The theorem of Ambrose-Singer [1] implies that the holonomy group of an affinely connected (resp. riemannian, kahlerian) manifold is automatically an algebraic (resp. riemannian, kahlerian) holonomy group. The sufficient conditions for the converse to be true are not known.

Definition 3. A triple $\{V, R, H\}$ is called a symmetric holonomy system if and only if $R$ is a curvature tensor on $V$ and $H$ is a connected Lie subgroup of $G Y(V)$ such that,

$$
\begin{gathered}
\mathfrak{h}=\operatorname{span}\left\{R_{x y}: x, y \in V\right\} \\
R_{h(x) y}+R_{x h(y)}+\left[R_{x y}, h\right]=0, \quad \forall h \in \mathfrak{h}, x, y \in V .
\end{gathered}
$$

It is called a riemannian (resp. kahlerian) symmetric holonomy system if and only if $R$ is furthermore a riemannian (resp. kahlerian) curvature tensor.

REMARK 2. The epithet "algebraic" has been dropped in this definition because we shall show in the next section that every such $H$ can actually be realized as the holonomy group of an appropriate symmetric space.

REMARK 3. Condition (7) corresponds to the requirement in the geometric situation that parallel translation of curvature is constant along closed paths, or equivalently, that differentiation of the curvature matrix function on the holonomy bundle (Section 2 of [15]) by a vertical vector field is zero. It is well-known that every affine (resp. riemannian, hermitian) symmetric space gives rise to a symmetric (resp. riemannian, kahlerian) holonomy system as follows: Consider an arbitrary tangent space $M_{m}$ of the manifold $M$ at $m$, and let the curvature tensor 
field evaluated at $m$ be $R$, and let $H$ be the holonomy group at $m$. Then $\left\{M_{m}, R, H\right\}$ is the required object. For a proof of [7] or [10]; (6) is implied by the Ambrose-Singer Theorem.

3. In preparation for the proofs of Theorems 2, 3, and 5, we collect together here all the facts we need about symmetric spaces. Basic for us is the following generalization of a theorem of Cartan's due to Nomizu. It is an immediate consequence of formula (9.6) and Theorems 13.1, 8.1, 10.2, 10.3 of [13].

Proposition 1. Let $G / K$ be a homogeneous space with $G$ connected and $K$ closed in $G$, such that the Lie algebras $\mathfrak{g}$ and $\mathfrak{f}$ of $G$ and $K$ admit a direct sum representation $\mathfrak{g}=\mathfrak{l} \oplus \mathfrak{m}$, where $\mathfrak{m}$ is a vector space, $[\mathfrak{m}, \mathfrak{m}] \leqq \mathfrak{l}$ and $[\mathfrak{f}, \mathfrak{m}] \leqq \mathfrak{m}$. Then there exists a (unique) canonical connection on $G / K$ such that it is torsionless and the covariant differential of its curvature tensor $R$ is zero. This connection is complete. Furthermore, if we identify the tangent space of $G / K$ at the coset $H$ with $\mathfrak{m}$, then $R$ satisfies:

$$
R_{x y} z=[[x, y], z], \quad \forall x, y, z \in \mathfrak{m}
$$

where the bracket is taken in $\mathfrak{g}$. Suppose in addition that on $\mathfrak{m}$ is defined an inner product $Q$ which is invariant under $A d_{\mathrm{m}}(K)$, then the riemannian connection of the left invariant metric on $G / K$ induced by $Q$ coincides with this canonical connection.

Since $G / K$ is complete in this connection, a standard application of the Ambrose-Hicks Theorem ([9], Theorem 1, p. 224) yields:

COROLLARY 1. The universal covering manifold of $G / K$ in the canonical connection of Proposition 1 is an affine symmetric space. This symmetric space is riemannian if $A d(K)$ leaves invariant an inner product on $\mathrm{m}$.

On the basis of this, it is easy to manufacture symmetric spaces with prescribed holonomy group. Thus, take a symmetric holonomy system $\{V, R, H\}$ and we form the vector space $\mathfrak{g}=\mathfrak{h} \oplus V$. Define a linear map $[]:, \mathfrak{g} \wedge \mathfrak{g} \rightarrow \mathfrak{g}$ by:

$$
\begin{cases}{\left[h_{1}, h_{2}\right]=\text { bracket in } \mathfrak{h}} & \text { if } h_{1}, h_{2} \in \mathfrak{h} \\ {\left[h_{1}, x\right]=h_{1}(x)} & \text { if } h_{1} \in \mathfrak{h}, x \in V \\ {[x, y]=R_{x y}} & \text { if } x, y \in V .\end{cases}
$$

This $[$,$] satisfies the Jacobi identity because of (7). Thus, g$ becomes a Lie algebra with $[V, V]=\mathfrak{h}$ (in view of $(6)$ ) and $[\mathfrak{h}, V] \subseteq V$. Now 
take a simply connected group $G$ with Lie algebra $g$ and let $H^{r}$ be the connected subgroup of $G$ corresponding to $\mathfrak{h}$. On $\mathfrak{g}$ is defined a Lie algebra involution $\sigma: h+x \rightarrow h-x$ where $h \in \mathfrak{h}$ and $x \in V$. This involution induces an involution of the group $G$, which we also denote by $\sigma$. It is clear then that $H^{r}$ is the identity component of the fixed point set of $\sigma$ in $G$ has hence is closed. Furthermore, $G / H^{r}$ is simply connected; one may see this either by elementary arguments or by the fibre homotopy sequence. Finally if $R$ is riemannian, then (2) implies that $\langle$,$\rangle on V$ is $A d\left(H^{r}\right)$-invariant. Corollary 1 therefore applies and $M=G / H^{r}$ is an affine or riemannian symmetric space in a natural way, depending on whether or not $R$ is riemannian. In addition (8) and $[V, V]=\mathfrak{h}$ imply that $a d_{v}(\mathfrak{h})$ is the holonomy algebra, which by definition 3 and formula (9), is just $\mathfrak{h}$ (where, now, the latter is again considered as a subalgebra of $\operatorname{gl}(V)$ ). So the holonomy group $A d_{v}\left(H^{r}\right)$ is just $H$. Summarizing, we have:

Corollary 2. If $\{V, R, H\}$ is a symmetric (resp. riemannian) holonomy system, then there exists a simply connected affine (resp. riemannian) symmetric space whose tangent space at a point can be identified with $V$, whose curvature tensor is $R$, and whose holonomy. group is $H$.

We want to derive another consequence of this general method of construction. If $M$ is an arbitrary simply connected affine symmetric space, then we have seen (Remark 3 of Section 2 ) that one can associate with $M$, in a canonical way, a symmetric holonomy system: $\left\{M_{m}, R, H\right\}$ where $H$ is the holonomy group of $M$. The remarks preceding Corollary 2 show the existence of a coset space $G / K$ which is simply connected and which has $R$ for its curvature tensor. Since $G / K$ and $M$ are both symmetric and have the same curvature tensor at a point, one may apply the Ambrose-Hicks Theorem to deduce that $G / K$ and $M$ are affinely diffeomorphic. We identify $M$ and $G / K$ via this diffeomorphism. Thus we may express the remarks preceding Corollary 2 in yet another way:

COROLLARY 3. Every simply connected affine symmetric space $M$ can be expressed as a "coset-space in reduced form," i.e. $M=G / K$, where $K \leqq G$ are connected Lie groups, $K$ is the identity component of the fixed point set of a certain involution $\sigma$ of $G$, and furthermore, if $\mathfrak{g}=\mathfrak{t} \oplus \mathfrak{p}, \mathfrak{p}$ being the subspace of $\mathfrak{g}$ which is the (-1)-eigenspace of $(d \sigma)_{e}(e=i d e n t i t y)$ on $\mathfrak{g}$, then $[\mathfrak{p}, \mathfrak{p}]=\mathfrak{k}$. Consequently, the holonomy group of $M$ is $A d_{p}(K)$.

REMARKS 4. The significance of this corollary lies in the assertion 
of the equality: $[\mathfrak{p}, \mathfrak{p}]=\mathfrak{f}$. In general, one has only inclusion in an arbitrary coset space representation of $M$.

4. We proceed to give the proofs of Theorem 2 and the assertions of Example 3 in this section. First, we need a simple fact:

Proposition 2. Let $G$ be a simply-connected solvable Lie group and $K$ an arbitrary connected Lie subgroup of $G$. Thus $G / K$ is diffeomorphic to a euclidean space.

Proof. It is a well-known theorem of Chevalley [6] that under the hypothesis of the theorem, $G$ is diffeomorphic to a euclidean space and $K$ can be represented as a global slice in $G$, i.e., there exists a global coordinate system $\left\{x_{1}, \cdots, x_{d}\right\}$ on $G$ such that $K=\{k: k \in G$, $\left.x_{p+1}(k)=\cdots=x_{d}(k)=0\right\}$. From this the proposition is immediate.

Proof of Theorem 2. Let $\mathrm{g}^{*}$ be the vector space dual of $g$, then the adjoint representation of $g$ into itself defines a dual representation $\varphi$ of $\mathfrak{g}$ in $\mathfrak{g}^{*}$ :

$$
\left\{\phi\left(g_{1}\right) g^{*}\right\}\left(g_{2}\right)=-g^{*}\left(\left[g_{1}, g_{2}\right]\right), \quad \forall g_{1}, g_{2} \in \mathfrak{g}, g^{*} \in \mathfrak{g}^{*} .
$$

Give $g^{*}$ the structure of an abelian Lie algebra and let $g^{\prime}$ be the semidirect product $\mathfrak{g} \ominus \mathfrak{g}^{*}$ of $\mathfrak{g}$ and $\mathfrak{g}^{*}$ relative to $\varphi$ i.e., $\left[\left(g_{1}, g_{1}^{*}\right),\left(g_{2}, g_{2}^{*}\right)\right]=\left(\left[g_{1}, g_{2}\right], \varphi\left(g_{1}\right) g_{2}^{*}-\varphi\left(g_{2}\right) g_{1}^{*}\right) \quad \forall g_{1}, g_{2} \in \mathfrak{g}, g_{1}^{*}, g_{2}^{*} \in \mathfrak{g}^{*}$.

Following Bourbaki ([5], p. 131), we shall introduce an $\operatorname{ad}\left(\mathfrak{g}^{\prime}\right)$-invariant inner product on $\mathrm{g}^{\prime}$ as follows:

$$
\left\langle\left(g_{1}, g_{1}^{*}\right),\left(g_{2}, g_{2}^{*}\right)\right\rangle=g_{1}^{*}\left(g_{2}\right)+g_{2}^{*}\left(g_{1}\right) .
$$

It is clear that this $\langle$,$\rangle is both nondegenerate and bilinear. (Its$ signature is $(d, d), d=\operatorname{dim} g$.$) One verifies easily that:$

$$
\begin{aligned}
& \left\langle\left\{a d\left(g, g^{*}\right)\right\}\left(g_{1}, g_{1}^{*}\right),\left(g_{2}, g_{2}^{*}\right)\right\rangle \\
& \quad=g_{2}^{*}\left(\left[g, g_{1}\right]\right)-g_{1}^{*}\left(\left[g, g_{2}\right]\right)+g^{*}\left(\left[g_{1}, g_{2}\right]\right) \\
& \quad=-\left\langle\left(g_{1}, g_{1}^{*}\right),\left\{a d\left(g, g^{*}\right)\right\}\left(g_{2}, g_{2}^{*}\right)\right\rangle .
\end{aligned}
$$

Thus $<,>$ is $a d\left(\mathfrak{g}^{\prime}\right)$-invariant. Let $\mathfrak{a}=\mathfrak{g}^{\prime} \oplus \mathfrak{g}^{\prime}$ be the direct product of $\mathrm{g}^{\prime}$ with itself, then $<$, $>$ extends naturally to an inner product on $a$, which we shall continue to denote by $\langle$,$\rangle .$

By standard practice, we decompose $a$ into $a=\vartheta \oplus \vartheta$, where $\vartheta=\left\{(g, g): g \in \mathfrak{g}^{\prime}\right)$ and $\vartheta^{-}=\left\{(g,-g): g \in g^{\prime}\right\}$ are respectively the diagonal and skew diagonal of $\mathfrak{a}$ with respect to $\mathfrak{g}^{\prime}$. It is obvious that $[\vartheta, \vartheta] \subseteq \vartheta$, $\left[\vartheta^{-}, \vartheta^{-}\right] \subseteq \vartheta,\left[\vartheta, \vartheta^{-}\right] \subseteq \vartheta^{-}$, and that the inner product on $\vartheta^{-}$inherited 
from $a$ is $a d(\vartheta)$-invariant. But instead of considering $a$, we shall take only the subalgebra $a^{-}=\left[\vartheta^{-}, \vartheta^{-}\right] \oplus \vartheta^{-}$of a. (Cf. (c) of Section 7.) The space $M$ of the theorem will in reality be $A / D$ where $A$ is a simply connected Lie group with Lie algebra $a^{-}$, and $D$ is the connected subgroup of $A$ corresponding to $\left[\vartheta^{-}, \vartheta^{-}\right]$. For the moment, we try to obtain a nice decomposition of $a^{-}$. We note that $a^{-}$is a solvable algebra because $g$ is (and so are $g^{\prime}$ and $a$ ).

Let $\vartheta^{-}=\vartheta_{1}^{-} \oplus \cdots \oplus \vartheta_{-}^{-}$be a decomposition of $\vartheta^{-}$into mutually orthogonal nondegenerate subspaces such that each is left invariant by $\operatorname{ad}\left(\left[\vartheta^{-}, \vartheta^{-}\right]\right)$and on each of which, $\operatorname{ad}\left(\left[\vartheta^{-}, \vartheta^{-}\right]\right)$acts weakly irreducibly. Thus,

$$
\begin{aligned}
{\left[\vartheta^{-}, \vartheta^{-}\right] } & =\left(\oplus_{i \neq j}\left[\vartheta_{\imath}^{-}, \vartheta_{j}^{-}\right]\right) \oplus\left(\bigoplus_{m=1, \ldots, \mathfrak{r}}\left[\vartheta_{m}^{-}, \vartheta_{m}^{-}\right]\right) \\
& \equiv \mathfrak{c} \oplus\left(\oplus_{m=1, \ldots \mathfrak{r}}\left[\vartheta_{m}^{-}, \vartheta_{m}^{-}\right]\right) .
\end{aligned}
$$

Clearly the invariance of each $\vartheta_{i}^{-}$under $\operatorname{ad}\left(\left[\vartheta^{-}, \vartheta^{-}\right]\right)$implies that $c$ and each $\left[\vartheta_{m}^{-}, \vartheta_{m}^{-}\right]$is a subalgebra. We claim:

$$
a d(c) \text { acts trivially on } \vartheta \text {. }
$$

$$
\text { If } m \neq n, a d\left(\left[\vartheta_{m}^{-}, \vartheta_{m}^{-}\right]\right) \text {acts trivially on } \vartheta_{n}^{-} \text {. }
$$

To prove (10), first note that $\left\langle a d\left(\left[d_{i}, d_{j}\right]\right) d_{m}, d_{n}\right\rangle=\left\langle a d\left(\left[d_{m}, d_{n}\right]\right) d_{\imath}, d_{j}\right\rangle$ for all $i, j, m, n$, and for $d_{\alpha} \in \vartheta_{\alpha}^{-}, \alpha=i, j, m, n$. This can be proved the same way that (4) of Section 2 is proved on the basis of (1) and (2). So, symbolically, one may write for $i \neq j$ that

$$
\left\langle\operatorname{ad}\left(\left[\vartheta_{\bar{i}}^{-}, \vartheta_{j}^{-}\right]\right) \vartheta_{m}^{-}, \vartheta_{n}^{-}\right\rangle=\left\langle\operatorname{ad}\left(\left[\vartheta_{m}^{-}, \vartheta_{n}^{-}\right]\right) \vartheta_{i}^{-}, \vartheta_{j}^{-}\right\rangle=0 .
$$

The last equality is because each $\vartheta_{i}^{-}$is invariant under $a d\left(\left[\vartheta^{-}, \vartheta^{-}\right]\right)$and because the $\vartheta_{\imath}^{-}$are assumed orthogonal to each other. Since $n$ is arbitrary, the above implies that $\left\langle a d(c) \vartheta_{m}^{-}, \vartheta^{-}\right\rangle=0$, which in turn implies that $\operatorname{ad}(\mathrm{c}) \vartheta_{m}^{-}=0$ because $\langle$,$\rangle is nondegenerate. The proves (10)$. For (11), note that by Jacobi identity:

$$
\left\langle\alpha d\left(\left[\vartheta_{m}^{-}, \vartheta_{m}^{-}\right]\right) \vartheta_{n}^{-}, \vartheta_{n}^{-}\right\rangle=\left\langle-a d\left(\left[\vartheta_{m}^{-}, \vartheta_{n}^{-}\right]\right) \vartheta_{m}^{-}, \vartheta_{n}^{-}\right\rangle+\left\langle-a d\left(\left[\vartheta_{n}^{-}, \vartheta_{m}^{-}\right]\right) \vartheta_{m}^{-}, \vartheta_{n}^{-}\right\rangle .
$$

Using invariance, $\operatorname{ad}\left(\left[\vartheta_{m}^{-}, \vartheta_{n}^{-}\right]\right) \vartheta_{m}^{-} \subseteq \vartheta_{m}^{-}$; since $m \neq n$, the right side vanishes, again because of mutual orthogonality of the $\vartheta_{i}^{-1}$ s. Since $<,>$ is nondegenerate on $\vartheta_{n}, \operatorname{ad}\left(\left[\vartheta_{m}, \vartheta_{m}\right]\right) \vartheta_{n}^{-}=0$. So (11) is also proved.

We have therefore shown that:

$$
\begin{aligned}
\mathfrak{a}^{-} & =\left(\mathfrak{c} \oplus\left\{\bigoplus_{m=1}, \ldots, \mathfrak{l}\left[\vartheta_{m}^{-}, \vartheta_{m}^{=}\right]\right\}\right) \oplus\left(\vartheta_{1}^{-} \oplus \cdots \oplus \vartheta_{-}^{-}\right) \\
& =\mathfrak{c} \oplus\left(\left[\vartheta_{1}^{-}, \vartheta_{1}^{-}\right] \oplus \vartheta_{1}\right) \oplus \cdots \oplus\left(\left[\vartheta_{\mathfrak{l}}^{-}, \vartheta_{\mathfrak{l}}^{-}\right] \oplus \vartheta_{\mathfrak{l}}^{-}\right)
\end{aligned}
$$

with the property that $a d(c)$ acts trivially on each $\vartheta_{i}^{-}$to its right, and that $\operatorname{ad}\left(\left[\vartheta_{i}^{-}, \vartheta_{i}^{-}\right]\right)$acts trivially on $\vartheta_{j}^{-}$if $i \neq j$. We now perform a final 
reduction on $\mathfrak{a}^{-}$. Let us group together all the $\vartheta_{i}^{-\prime} \mathrm{s}$ such that $\operatorname{ad}\left(\left[\vartheta_{i}^{-}, \vartheta_{i}^{-}\right]\right) \vartheta_{i}^{-}=0$, say, these are the first $q$ of them. Let $\mathfrak{\mathfrak { f }}_{0}=$ $\mathfrak{c} \oplus\left\{\oplus_{i=1, \ldots, q}\left[\vartheta_{i}^{-}, \vartheta_{i}^{-}\right]\right\}$, and $\mathfrak{p}_{0}=\vartheta_{1}^{-} \oplus \cdots \oplus \vartheta_{q}^{-}$. For the remaining $\vartheta_{\imath}^{-\prime} \mathbf{s}$ (IF there are any left), we shall relabel them to be $\mathfrak{p}_{1}, \cdots, \mathfrak{p}_{k}$, and shall denote $\left[\vartheta_{j}^{-}, \vartheta_{j}^{-}\right]$by $\mathfrak{f}_{j}$. We have finally obtained:

The solvable algebra $a^{-}=\left[\vartheta^{-}, \vartheta^{-}\right] \oplus \vartheta^{-}$can be expressed as a direct sum of its subalgebras:

$$
\mathfrak{a}^{-}=\mathfrak{g}_{0} \oplus \cdots \oplus \mathfrak{g}_{k}
$$

where: $\mathfrak{g}_{0}=\mathfrak{l}_{0} \oplus \mathfrak{p}_{0},\left[\mathfrak{p}_{0}, \mathfrak{p}_{0}\right]=0, \operatorname{ad}\left(\mathfrak{\mathfrak { t }}_{0}\right) \mathfrak{p}_{0}=0$ and for $i \geqq 1$,

$$
\mathfrak{g}_{i}=\mathfrak{\mathfrak { t }}_{i} \oplus \mathfrak{p}_{i}, \mathfrak{\mathfrak { t }}_{i}=\left[\mathfrak{p}_{i}, \mathfrak{p}_{i}\right], 0 \neq a d\left(\mathfrak{f}_{i}\right) \mathfrak{p}_{i} \subseteq \mathfrak{p}_{i} .
$$

Furthermore:

$$
\begin{aligned}
a d\left(\mathfrak{k}_{j}\right) \mathfrak{p}_{i}=0 & \text { if } \quad i \neq j \\
{\left[\vartheta^{-}, \vartheta^{-}\right]=\mathfrak{k}_{0} \oplus \mathfrak{l}_{1} \oplus \cdots \oplus \mathfrak{t}_{k}, } & \vartheta^{-}=\mathfrak{p}_{0} \oplus \mathfrak{p}_{1} \oplus \cdots \oplus \mathfrak{p}_{k} .
\end{aligned}
$$

We are now in a position to apply Proposition 1 to construct the required symmetric spaces. For each $\mathrm{g}_{\alpha} \alpha=0, \cdots, k$, take a simply connected group $G_{\alpha}$ whose Lie algebra is $\mathfrak{g}_{\alpha}$, and let $K_{\alpha}$ be the connected subgroup corresponding to $\mathfrak{f}_{\alpha}$. Since $\mathfrak{a}^{-}$is solvable, $\mathfrak{g}_{\alpha}$ and therefore $G_{\alpha}$ is solvable for each $\alpha$. Thus Proposition 2 tells us that each $G_{\alpha} / K_{\alpha}$ is diffeomorphic to a euclidean space. Corollary 1 of Section 3 applies. So, letting $M_{\alpha}=G_{\alpha} / K_{\alpha}$, formula (8) implies the curvature tensor of $M_{0}$ is null, while the curvature transformations of each $M_{i}, i \geqq 1$, acts weakly irreducibly by virtue of (12). Since the curvature transformations span the holonomy algebra, the holonomy group of each $M_{i}$ is weakly irreducible and we have the existence of a representation $M$ as the product of a flat factor and a finite number of weakly irreducible symmetric spaces. We remark that this product decomposition is an immediate consequence of the de Rham Decomposition Theorem [15], and we need not have obtained (12) and (13) first. But for the other assertions, it is convenient to have the latter, as we shall see right away.

First, we settle the existence of the $M_{i}$, for $i \geqq 1$, under the hypothesis that $[\mathfrak{g}, \mathscr{D} \mathfrak{g}] \neq 0$. (Thus we are proving $[\mathfrak{g}, \mathscr{D} \mathfrak{g}] \neq 0 \Rightarrow k \geqq 1$.) This is equivalent to showing $k \geqq 1$ in the decomposition (12) of $a^{-}$. Now, $[g, \mathscr{D g}] \neq 0 \Rightarrow\left[g^{\prime}, \mathscr{D} g^{\prime}\right] \neq 0 \Rightarrow[\vartheta,[\vartheta, \vartheta]] \neq 0$, all of those implications being trivial from the relevant definitions. By (13), we have then, $\Theta_{i=1, \ldots, k}\left[\mathfrak{p}_{i}, \mathfrak{f}_{i}\right] \neq 0$. By (12), this is equivalent to $k \geqq 1$.

If now $\left[\mathfrak{g}, \mathscr{D}^{2} \mathrm{~g}\right] \neq 0$, then one obtains as above that $\left[\vartheta^{-}, \mathscr{D}\left(\left[\vartheta^{-}, \vartheta^{-}\right]\right)\right] \neq 0$. By (13) and the Jacobi identity, it is clear that, $\bigoplus_{i=0, \ldots, k}\left[\mathfrak{p}_{i},\left[\mathfrak{f}_{i}, \mathfrak{k}_{i}\right]\right] \neq 0$, or equivalently, $\left.0 \neq \bigoplus_{i=1, \ldots, k} a d_{\mathfrak{F}_{i}} \mathfrak{\mathfrak { k }}_{i}, \mathfrak{\mathfrak { l }}_{i}\right], \quad$ or 
equivalently, $0 \neq \bigoplus_{i=1, \ldots, k}\left[a d_{\mathfrak{p}_{i}} \mathfrak{f}_{i}, a d_{\mathfrak{p}_{i}} \mathfrak{f}_{i}\right]$. But $\mathfrak{\mathfrak { f }}_{i}=\left[\mathfrak{p}_{i}, \mathfrak{p}_{i}\right]$ (by (12)), and (8) therefore implies that $a d_{\mathfrak{p}_{i}}\left(\mathfrak{f}_{i}\right)$ is precisely the holonomy algebra of $M_{i}, i \geqq 1$. So the holonomy algebra, and hence the holonomy group, of at least one of the $M_{i}^{\prime} \mathrm{s}$ is nonabelian. It is however solvable because $a^{-}$is, and consequently so is each $\mathfrak{k}_{i}, i=1, \cdots, k$.

Now, we shall show that in the event that $k \geqq 1$, all $M_{i}, i=1$, $\cdots, k$, are $S-W$ irreducible. Suppose not, then at least one of them, say, $M_{\beta}$ is irreducible in the usual sense; for, by definition, each $M_{i}$ is weakly irreducible to start with. But then by a theorem of Nomizu [13], $\left[\mathfrak{p}_{\beta}, \mathfrak{p}_{\beta}\right]=\mathfrak{l}_{\beta} \neq 0 \Longrightarrow \mathfrak{g}_{\beta}$ is semisimple. This contradicts the solvability of the $\mathfrak{g}_{i}^{\prime} \mathrm{s}$.

Finally we prove that the matrix algebra $\operatorname{st}(d)$ of $d \times d$ supertriangular matrices with zero trace gives rise to a $S-W$ irreducible manifold $M$. As usual denote the vector space dual of $\mathfrak{s t}(d)$ by $\operatorname{st}(d)^{*}$ and let $\mathfrak{g}=\operatorname{st}(d) \Theta \mathfrak{s t}(d)^{*}$ (s.d. product). By the above construction of the manifold, (especially (12) and (13)), it suffices to prove that $\mathscr{D} \mathfrak{g}$ acts weakly irreducibly on $\mathfrak{g}$ via the adjoint representation. We shall do that, and even a little bit more.

Let $\varepsilon_{i j}$ denote the $d \times d$ matrix whose $(i, j)$-th entry equals one, and all other entries equal zero. In the rest of this proof, we adhere to the convention that all $\varepsilon_{i j}$ 's considered will have the property that $i<j$, and that the diagonal matrices will be explicitly given as $\varepsilon_{i i}$. Thus, at $(d)=\left\{\varepsilon_{i j}, \sum_{i} \alpha_{i} \varepsilon_{i i}: \sum_{i} \alpha_{i}=0, \alpha_{i} \in \boldsymbol{R}\right.$, all $\left.i, j\right\}$. The dual basis in $\stackrel{s t}{t}(d)^{*}$ of the $\varepsilon_{i j}$ and $\sum_{i} \alpha_{i} \varepsilon_{i i}$ are defined by:

$$
\begin{aligned}
& \varepsilon_{k \mathfrak{l}}^{*}\left(\varepsilon_{i j}\right)=\delta_{l i} \delta_{\mathfrak{l}} \\
& \varepsilon_{i i}^{*}\left(\varepsilon_{j j}\right)=\delta_{i j} .
\end{aligned}
$$

So, with the same convention on the indices of $\varepsilon_{i j}^{*}$ as above: $\operatorname{st}(d)^{*}=$ $\left\{\varepsilon_{i j}^{*}, \sum_{i} \alpha_{i} \varepsilon_{i i}^{*}: \sum_{i} \alpha_{i}=0, \alpha_{i} \in \boldsymbol{R}\right.$, all $\left.i, j\right\}$.

To further simplify matters, $\left(\varepsilon_{i j} \varepsilon_{k \mathrm{l}}^{*}\right)$ etc. will denote the transform of $\varepsilon_{k r}^{*}$ by $\varepsilon_{i j}$ under the dual of the adjoint representation. We also frequently write $\varepsilon_{i j}, \varepsilon_{i j}^{*}$, etc. in place of $\left(\varepsilon_{i j}, 0\right),\left(0, \varepsilon_{i j}^{*}\right)$ etc. as elements of $\mathfrak{g}$. In other words, we identify $\mathfrak{s t}(d)$ with $(\mathfrak{s t}(d), 0)$ of $\mathfrak{g}$, and $\mathfrak{s t}(d)^{*}$ with $\left(0, \mathfrak{s t}(d)^{*}\right)$ of $\mathfrak{g}$. The following summarizes all bracket operations in $\mathrm{g}$ :

$$
\begin{aligned}
& {\left[\varepsilon_{i j}, \varepsilon_{k \mathfrak{l}}\right]=\delta_{j k} \varepsilon_{i \mathfrak{}}-\delta_{i} \varepsilon_{k j}} \\
& {\left[\varepsilon_{i i}, \varepsilon_{i j}\right]=\varepsilon_{i j}} \\
& {\left[\varepsilon_{i i}, \varepsilon_{j j}\right]=0} \\
& {\left[\varepsilon_{i j}^{*}, \varepsilon_{k \mathfrak{l}}^{*}\right]=0} \\
& {\left[\varepsilon_{\mathfrak{l l}}, \varepsilon_{\mathfrak{l} j}^{*}\right] \equiv \varepsilon_{\mathfrak{l} l} \varepsilon_{\mathfrak{l}}^{*} \varepsilon_{\mathfrak{l}}^{*}=-\varepsilon_{\mathfrak{l} j}^{*}} \\
& {\left[\varepsilon_{\mathfrak{l l}}, \varepsilon_{j \mathfrak{l}}^{*}\right] \equiv \varepsilon_{\mathfrak{l l}} \varepsilon_{\mathfrak{j} \mathfrak{l}}^{*}=\varepsilon_{\mathfrak{l} j}^{*}}
\end{aligned}
$$




$$
\begin{aligned}
& {\left[\varepsilon_{i j}, \varepsilon_{k \mathfrak{l}}^{*}\right] \equiv \varepsilon_{i j} \varepsilon_{k \mathfrak{l}}^{*}=0 \text { if } i \neq k, j \neq \mathfrak{l}}
\end{aligned}
$$

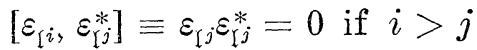

$$
\begin{aligned}
& {\left[\varepsilon_{\mathfrak{l}}, \varepsilon_{\mathfrak{l} j}^{*}\right] \equiv \varepsilon_{\mathfrak{l}} \varepsilon_{\mathfrak{l} j}^{*}=-\varepsilon_{i j}^{*} \text { if } i<j}
\end{aligned}
$$

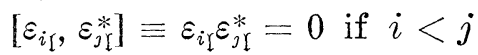

$$
\begin{aligned}
& {\left[\varepsilon_{i \mathfrak{l}}, \varepsilon_{j \mathfrak{j}}^{*}\right] \equiv \varepsilon_{i \mathfrak{l}} \varepsilon_{i j}^{*}=\varepsilon_{j \mathfrak{l}}^{*} \text { if } i>j} \\
& {\left[\varepsilon_{i j}, \varepsilon_{\mathfrak{I}}^{*}\right] \equiv \varepsilon_{i j} \varepsilon_{\mathfrak{I}}^{*}=0} \\
& {\left[\varepsilon_{i j}, \varepsilon_{i j}^{*}\right] \equiv \varepsilon_{i j} \varepsilon_{i j}^{*}=\varepsilon_{i i}^{*}-\varepsilon_{j j}^{*} \text {. }}
\end{aligned}
$$

We shall make extensive computations on the basis of this set of formulae and shall not explicitly refer to them again. The first trivial consequence of this is:

$$
\mathscr{D} \mathfrak{g}=\left\{\varepsilon_{i j}, \varepsilon_{i j}^{*}, \sum_{i} \alpha_{i} \varepsilon_{i i}^{*}: \alpha_{i} \in \boldsymbol{R}, \sum_{i} \alpha_{i}=0 \text {, all } i, j\right\} \text {. }
$$

Now assume $\mathfrak{g}=\mathfrak{p} \oplus \mathfrak{q}$, where $\mathfrak{p}, \mathfrak{q}$ are vector spaces such that:

$$
\text { (*) } \quad \mathfrak{p}, \mathfrak{q} \text { are invariant under } \operatorname{ad}(\mathscr{D} \mathfrak{g}) \text {. }
$$

(From now on, ad will denote the adjoint representation of g.)

$$
\begin{aligned}
(* *) & \mathfrak{p} \cap \mathfrak{q}=\{0\} \\
(* * *) & \langle\mathfrak{p}, \mathfrak{q}\rangle=0 .
\end{aligned}
$$

On the strength of these a assumptions alone, we shall prove that one of $\mathfrak{p}$ and $q$ must be zero. This is clearly stronger than the statement that $g$ is weakly irreducible under $\operatorname{ad}(\mathscr{D} \mathfrak{g})$, so that once this is done, we have proved the theorem. The crux of the matter is contained in the

Lemma. If $\mathfrak{g}=\mathfrak{p} \oplus \mathfrak{q}$ such that $\left({ }^{*}\right)-\left({ }^{* * *}\right)$ are satisfied, then all elements of the form $\varepsilon_{i i}-\varepsilon_{j j}, i<j$, are either in $\mathfrak{p}$ or in $\mathfrak{q}$.

We first prove this lemma. Since the case $d=2$ is quite easy to verify directly, we assume henceforth that $d \geqq 3$. For the moment, consider $\varepsilon_{11}-\varepsilon_{22}$ and suppose

$$
\begin{aligned}
\varepsilon_{11}-\varepsilon_{22}= & \left(\left(\sum_{i=1}^{d} a_{i} \varepsilon_{i i}\right)+\sum_{i, j} \alpha_{i j} \varepsilon_{i j}, \sum_{i=1}^{d} b_{i} \varepsilon_{i i}^{*}+\sum_{i j} \beta_{i j} \varepsilon_{i j}^{*}\right) \\
& \oplus\left(\left(1-a_{1}\right) \varepsilon_{11}+\left(-1-a_{2}\right) \varepsilon_{22}-\sum_{i \geq 3} a_{i} \varepsilon_{i i}-\sum_{i j} \alpha_{i j} \varepsilon_{i j},\right. \\
& \left.-\sum_{i=1}^{d} b_{i} \varepsilon_{i i}^{*}-\sum_{i, j} \beta_{i j} \varepsilon_{i j}^{*}\right) \\
\equiv & A \oplus B
\end{aligned}
$$

where $A \in \mathfrak{p}, B \in \mathfrak{q}, \sum_{i=1}^{d} a_{i}=0, \sum_{i=1}^{d} b_{i}=0$. Of course, we aim at proving that all $\alpha_{i j}, \beta_{i j}, b_{i}$, and all $\alpha_{i}$ for $i \geqq 3$, in fact vanish. First 
note that if $v=\sum_{i} x_{i} \varepsilon_{i i}, x_{i} \in R$, then $\left(a d \varepsilon_{m n}\right)(v)=\left(-x_{m n}+x_{n n}\right) \varepsilon_{m n}$. Let now $m, n \geqq 3$, then

$$
\begin{aligned}
& \left(a d \varepsilon_{m n}\right)(A)=\left(\left(-a_{m m}+a_{n n}\right) \varepsilon_{m n}+\left(a d \varepsilon_{m n}\right)(g),\left(a d \varepsilon_{m n}\right)\left(h^{*}\right)\right) \\
& \left(a d \varepsilon_{m n}\right)(B)=\left(\left(a_{m m}-a_{n n}\right) \varepsilon_{m n}-\left(a d \varepsilon_{m n}\right)(g),\left(a d \varepsilon_{m n}\right)\left(h^{*}\right)\right)
\end{aligned}
$$

where $g=\sum_{i, j} \varepsilon_{i j}, h^{*}=\sum_{l=1}^{d} b_{i} \varepsilon_{i i}^{*}+\sum_{i, j} \beta_{i j} \varepsilon_{i j}^{*}$. By virtue of (**), we conclude that: $\forall m, n \geqq 3, a_{m m}=a_{n n},\left(a d \varepsilon_{m n}\right)(g)=0,\left(a d \varepsilon_{m n}\right)\left(h^{*}\right)=0$. The second relation implies, as a little reflection will show, that if $i, j \geqq 3, \alpha_{i j}=0$ with the exception of $\alpha_{3 d}$, and the third relation implies that if $i, j \geqq 3, \beta_{i j}=0$ without exceptions. So we may rewrite:

$$
\varepsilon_{11}-\varepsilon_{22}=A \oplus B \text { relative to } \mathfrak{p} \oplus \mathfrak{q}
$$

where

$$
\begin{aligned}
A= & \left(a_{1} \varepsilon_{11}+a_{2} \varepsilon_{22}-\frac{a_{1}+a_{2}}{d-2}\left(\varepsilon_{33}+\cdots+\varepsilon_{d d}\right)\right. \\
& \left.+\sum_{i=1,2} \alpha_{i j} \varepsilon_{i j}+\alpha_{3 d} \varepsilon_{3 d}, \sum_{i=1}^{d} b_{\imath} \varepsilon_{i i}^{*}+\sum_{i=1,2} \beta_{\imath \jmath} \varepsilon_{i j}^{*}\right) \\
B= & \left(\left(1-a_{1}\right) \varepsilon_{11}+\left(-1-a_{2}\right) \varepsilon_{22}+\frac{a_{1}+a_{2}}{d-2}\left(\varepsilon_{33}+\cdots+\varepsilon_{d d}\right)\right. \\
& \left.-\sum_{i=1,2} \alpha_{\imath \jmath} \varepsilon_{i \jmath}-\alpha_{3 d} \varepsilon_{3 d},-\sum_{i=1}^{d} b_{i} \varepsilon_{i i}^{*}-\sum_{i=1,2} \beta_{i j} \varepsilon_{i j}^{*}\right) .
\end{aligned}
$$

Clearly either $a_{1} \neq 0$ or $1-a_{1} \neq 0$ or both. For definiteness, let $a_{1} \neq 0$. Suppose $1-a_{1} \neq 0$, and we shall deduce a contradiction. Consider:

$$
\begin{aligned}
& A_{1} \equiv\left(a d \varepsilon_{13}\right)(A)=\left(\left(-a_{1}-\frac{a_{1}+a_{2}}{d-2}\right) \varepsilon_{13}+\alpha_{3 d} \varepsilon_{1 d},\left(a d \varepsilon_{13}\right)\left(h^{*}\right)\right) \\
& B_{1} \equiv\left(a d \varepsilon_{13}\right)(B)=\left(\left(a_{1}-1+\frac{a_{1}+a_{2}}{d-2}\right) \varepsilon_{13}-\alpha_{3 d} \varepsilon_{1 d},-\left(a d \varepsilon_{13}\right)\left(h^{*}\right)\right) \\
& A_{2} \equiv\left(a d \varepsilon_{12}\right)(A)=\left(\left(-a_{1}+a_{2}\right) \varepsilon_{12}+\left(\alpha_{23} \varepsilon_{13}+\cdots+\alpha_{2 d} \varepsilon_{i d}\right),\left(a d \varepsilon_{12}\right)\left(h^{*}\right)\right) \\
& B_{2} \equiv\left(a d \varepsilon_{12}\right)(B)=\left(\left(a_{1}-a_{2}-2\right) \varepsilon_{12}-\left(\alpha_{23} \varepsilon_{13}+\cdots+\alpha_{2 d} \varepsilon_{1 d}\right),-\left(a d \varepsilon_{13}\right)\left(h^{*}\right)\right) \\
& A_{3} \equiv\left(a d \varepsilon_{23}\right)(A)=\left(\left(a_{2}+1+\frac{a_{1}+a_{2}}{d-2}\right) \varepsilon_{23}-\alpha_{12} \varepsilon_{13}+\alpha_{3 d} \varepsilon_{2 d},\left(a d \varepsilon_{23}\right)\left(h^{*}\right)\right) \\
& B_{3} \equiv\left(a d \varepsilon_{23}\right)(B)=\left(\left(a_{2}+1+\frac{a_{1}+a_{2}}{d-2}\right) \varepsilon_{23}+\alpha_{12} \varepsilon_{13}-\alpha_{3 d} \varepsilon_{2 d},-\left(a d \varepsilon_{23}\right)\left(h^{*}\right)\right)
\end{aligned}
$$

where $h^{*}=\sum_{i=1}^{d} b_{i} \varepsilon_{i i}^{*}+\sum_{i=1,2} \beta_{i j} \varepsilon_{i j}^{*}$. Of course, $A_{i} \in \mathfrak{p}$ and $B_{i} \in \mathfrak{q}$, for $i=1,2,3$.

Suppose the coefficient of $\varepsilon_{13}$ in $A_{1}, B_{1}$ are both nonzero. Apply $\left(a d \varepsilon_{34}\right)$ to both $A_{1}$ and $B_{1}$, and we get that $\varepsilon_{14} \in \mathfrak{p} \cap \mathfrak{q}$, which is absurd. So at least one of the coefficients of $\varepsilon_{13}$ in $A_{1}, B_{1}$ is zero. 
Case 1. $-a_{1}-\left(a_{1}+a_{2}\right) /(d-2)=0$ or, $a_{1}-a_{2}=d a_{1}$. We again have to consider two sub-cases separately.

Case 1a. $d a_{1} \neq 2$. Then $-a_{1}+a_{2}=-d a_{1} \neq 0$ and $a_{1}-a_{2}-2=$ $d a_{1}-2 \neq 0$. So the coefficients of $\varepsilon_{12}$ in $A_{2}$ and $B_{2}$ are both nonzero. In this case, application of $\left(a d \varepsilon_{23}\right)$ to both $A_{2}, B_{2}$ gives that $\varepsilon_{13} \in \mathfrak{p} \cap \mathfrak{q}$, again impossible.

Case 1b. $d a_{1}=2$ or $a_{1}-a_{2}=2$. Then,

$$
\left(-a_{2}-\frac{a_{1}+a_{2}}{d-2}\right)=\frac{1}{d-2}(2 d-4) \neq 0
$$

because $d \geqq 3$.

$$
\left(a_{2}+1+\frac{a_{1}+a_{2}}{d-2}\right)=-1
$$

Hence the coefficients of $\varepsilon_{23}$ in $A_{3}, B_{3}$ are both nonzero. Now apply successively $\left(\alpha d \varepsilon_{12}\right)$ and $\left(\alpha d \varepsilon_{34}\right)$ to $A_{3}, B_{3}$ in that order, and we will get that $\varepsilon_{14} \in \mathfrak{p} \cap \mathfrak{q}$, contradiction.

We conclude that Case 1 is impossible.

Case 2. $a_{1}-1+\left(a_{1}+a_{2}\right) /(d-2)=0$ or $a_{1}-a_{2}=2-d\left(1-a_{1}\right)$. As before, we have to consider two distinct possibilities.

Case 2a. $\quad d\left(1-a_{1}\right) \neq 2$. So $a_{1}-a_{2} \neq 0$, and consequently the coefficient of $\varepsilon_{12}$ in $A_{2}$ is nonzero. The coefficient of $\varepsilon_{12}$ in $B_{2}=a_{1}$ $a_{2}-2=-\left(1-a_{1}\right) d \neq 0$ because $a_{1} \neq 1$, by assumption. Hence, the coefficients of $\varepsilon_{12}$ in both $A_{2}, B_{2}$ are nonzero, and a contradiction can be deduced as before

Case 2b. $d\left(1-a_{1}\right)=2$, or $a_{1}-a_{2}=0$. This implies:

$$
\begin{gathered}
-a_{2}-\frac{a_{1}-a_{2}}{d-2}=\frac{-d}{d-2} a_{1} \neq 0 \\
\left(a_{2}+1\right)+\frac{a_{1}+a_{2}}{d-2}=\frac{(d+1)}{d-2} a_{1} \neq 0 .
\end{gathered}
$$

So again, the coefficients of $\varepsilon_{23}$ in $A_{3}, B_{3}$ are nonzero and there is a contradiction, as the above shows.

Thus Case 2 is also impossible.

We have therefore proved that necessarily $a_{1}=1$. We now prove further that necessarily $a_{2}=-1$. Suppose the contrary and we consider two cases. 
Case 1. $a_{2}=1$. In this case, the coefficients of $\varepsilon_{13}$ in $A_{1}, B_{1}$ are respectively $-1-2 /(d-2)$ and $2 /(d-2)$, and are nonzero. Deduce a contradiction as above.

Case 2. $a_{2} \neq 1$. In this case, the coefficients of $\varepsilon_{12}$ in $A_{2}, B_{2}$ are nonzero, again impossible.

We have demonstrated $a_{1}=-a_{2}=1$. This permits us to rewrite $A$ and $B$ in a much neater form: $\varepsilon_{11}-\varepsilon_{22}=A \oplus B$ relative to $\mathfrak{p} \oplus \mathfrak{q}$ where

$$
\begin{aligned}
& A=\left(\varepsilon_{11}-\varepsilon_{22}+\sum_{i=1,2} \alpha_{i j} \varepsilon_{i j}+\alpha_{3 d} \varepsilon_{3 d}, \sum_{i=1}^{d} b_{i} \varepsilon_{i \imath}^{*}+\sum_{i=1,2} \beta_{i j} \varepsilon_{i j}^{*}\right) \\
& B=\left(-\sum_{i=12} \alpha_{i j} \varepsilon_{i j}-\alpha_{3 d} \varepsilon_{3 d},-\sum_{i=1}^{d} b_{i} \varepsilon_{i i}^{*}-\sum_{i=1,2} \beta_{i j} \varepsilon_{i j}^{*}\right) .
\end{aligned}
$$

We next prove $\alpha_{12}=0$. Consider $\left(a d \varepsilon_{12}^{*}\right)(A)=\left(0,-\varepsilon_{11} \varepsilon_{12}^{*}+\varepsilon_{22} \varepsilon_{12}^{*}\right)=2\left(0, \varepsilon_{12}^{*}\right)$. Then $\left(^{* *}\right) \Rightarrow 0=\left\langle\left(0, \varepsilon_{12}^{*}\right), B\right\rangle=-\alpha_{12}$. In like manner, one shows $\alpha_{23}, \alpha_{13}, \alpha_{24}, \alpha_{14} \ldots$ must all vanish, in this order; here, we insist on the order of doing things because we are appealing to the fact that: $\varepsilon_{k j} \varepsilon_{k \mathfrak{l}}^{*}=0$ if $j>\mathfrak{l}, \varepsilon_{i j} \varepsilon_{k j}^{*}=0$ if $i<k$. Now suppose $\beta_{12} \neq 0$, then $\left(a d \varepsilon_{12}\right)(B)=-\left(0, \beta_{12}\left(\varepsilon_{11}^{*}-\varepsilon_{12}^{*}\right)-\beta_{13} \varepsilon_{23}^{*}-\cdots-\beta_{1 d} \varepsilon_{2 d}^{*}\right) \equiv D$. Thus $\langle A, D\rangle=$ $-2 \beta_{12} \neq 0$, contradicting $(* * *)$. We next prove $\beta_{23}=0 . \quad\left(a d \varepsilon_{23}\right)(B)=$ $\left(-\alpha_{3 d} \varepsilon_{2 d},-\beta_{13} \varepsilon_{12}^{*}-\beta_{23}\left(\varepsilon_{22}^{*}-\varepsilon_{23}^{*}\right)+\beta_{24} \varepsilon_{34}^{*}+\cdots+\beta_{2 d} \varepsilon_{3 d}^{*}\right) \equiv E$. By $\quad(* * *)$, $0=\langle A, E\rangle=-\alpha_{3 d} \beta_{2 d}+\beta_{23}+\beta_{2 d} \alpha_{3 d}=\beta_{23}$. In a manner similar to the demonstration of $\beta_{23}=0$, one can prove $\beta_{24}, \beta_{14}, \beta_{25}, \beta_{15}, \cdots$ all vanish; here again the order of demonstration is important, for reasons similar to above.

Thus, we can write relative to $\mathfrak{p} \oplus \mathfrak{q}$ :

$$
\begin{aligned}
\varepsilon_{11}-\varepsilon_{22}= & \left(\varepsilon_{11}-\varepsilon_{22}+\alpha_{3 d} \varepsilon_{3 d}, \sum_{i=1}^{d} b_{i} \varepsilon_{i i}^{*}\right) \\
& \oplus\left(-\alpha_{3 d} \varepsilon_{3 d},-\sum_{i=1}^{d} b_{i} \varepsilon_{i 2}^{*}\right) .
\end{aligned}
$$

Suppose $\alpha_{3 d} \neq 0 . \quad\left(a d \varepsilon_{13}\right)(B)=\alpha_{3 d} \varepsilon_{1 d} \Rightarrow \varepsilon_{1 d} \in \mathfrak{q}, \Rightarrow \varepsilon_{11}^{*}-\varepsilon_{d d}^{*}=\left(\alpha d \varepsilon_{1 d}^{*}\right)\left(\varepsilon_{1 d}\right) \in \mathfrak{q}$, $\Rightarrow 0=\left\langle A, \varepsilon_{11}^{*}-\varepsilon_{d d}^{*}\right\rangle=1$, which is not right. So $\alpha_{3 d}=0$. We shall show finally that $b_{i}=0 \forall i$. For, $\varepsilon_{12}=1 / 2 \cdot\left(a d \varepsilon_{12}\right)(A) \in \mathfrak{p}$ and $\varepsilon_{23}=$ $-\left(a d \varepsilon_{23}\right)(A) \in \mathfrak{p}$. Once $\varepsilon_{12}, \varepsilon_{23}$ are in $\mathfrak{p},\left(^{*}\right)$ easily implies that $\varepsilon_{12}, \cdots$, $\varepsilon_{1 d}, \varepsilon_{23}, \cdots, \varepsilon_{2 d}$ are all in $\mathfrak{p}$. But then, $\left(a d \varepsilon_{i j}^{*}\right)\left(\varepsilon_{i j}\right)=-\varepsilon_{i i}^{*}+\varepsilon_{i j}^{*}$, for $i=1,2$ and for all $j$, are in $\mathfrak{p}$. Hence if $\sum_{i} \gamma_{i}=0, \gamma_{i} \in \boldsymbol{R}$, then $\sum_{i} \gamma_{i} \varepsilon_{i i}^{*} \in \mathfrak{p}$. So if $b_{i}$ are not all zero, $0 \neq \sum_{i} b_{i} \varepsilon_{i i}^{*} \in \mathfrak{p} \cap \mathfrak{q}$, which contradicts $\left({ }^{*}\right)$. Hence finally $\left(\varepsilon_{11}-\varepsilon_{22}\right) \in \mathfrak{p}$.

In the above proof, the indices 1 and 2 play no special role and it is therefore clear that one can prove $\varepsilon_{i i}-\varepsilon_{j j}$ is in either $\mathfrak{p}$ or $\mathfrak{q}$ for arbitrary $i$ and $j$ in exactly the same manner. The lemma is thereby proved. 
Once we have the lemma, the conclusion of the proof of the theorem is straightforward. Let $\mathfrak{g}=\mathfrak{p} \oplus \mathfrak{q}$ satisfying $\left({ }^{*}\right)-\left({ }^{* *}\right)$, and let $\left(\varepsilon_{11}-\varepsilon_{22}\right) \in \mathfrak{p}$. As we have seen in the previous paragraph, this necessitates the fact that: $\varepsilon_{1 j}, \varepsilon_{2 k}$ are in $\mathfrak{p}$ for all $j \geqq 2, k \geqq 3$. Now, by the lemma again, we may assume $\varepsilon_{22}-\varepsilon_{33}$ is in either $\mathfrak{p}$ or $\mathfrak{q}$. If it is in $\mathfrak{q}$, then one proves similarly that: $\left(^{*}\right) \Rightarrow \varepsilon_{2 k}, \varepsilon_{3}$, for all $k>3$, $\mathfrak{l} \geqq 4$, are in $\mathfrak{q}$. This contradicts that $\varepsilon_{2 k}$ are already in $\mathfrak{p}$. Hence $\left(\varepsilon_{22}-\varepsilon_{33}\right) \in \mathfrak{p}$. We can continue this process and conclude that $\left(\varepsilon_{i i}-\varepsilon_{j j}\right)$ for all $i, j$ are in $\mathfrak{p}$. This means $\left\{\varepsilon_{i j}\right\} \subseteq \mathfrak{p}$, for all $i, j$. Since $\left(a d \varepsilon_{i j}^{*}\right)\left(\varepsilon_{i j}\right)=-\varepsilon_{i i}^{*}-\varepsilon_{i j}^{*}$, and $\left(a d \varepsilon_{i j}^{*}\right)\left(\varepsilon_{i k}\right)=\varepsilon_{i k}^{*}$ for $j<k,\left(^{*}\right)$ implies that all $\left\{\varepsilon_{i j}^{*}, \varepsilon_{k k}^{*}-\varepsilon_{\mathfrak{l}}^{*}\right)$ are also in $\mathfrak{p}$. Hence $\mathfrak{p}=\{0\}$.

REMARK 5. It is easy to see that the matrix algebra $t(d)$ of all triangular matrices is the direct sum of two ideals: span $\left\{\varepsilon_{11}+\cdots+\varepsilon_{d d}\right\}$ and $\operatorname{st}(d)$. Correspondingly, the manifold associated with $t(d)$ is isometric to the direct product of the Lorentz plane and the manifold associated with $s t(d)$. This is a special case of a general fact, namely, that if the algebra $g$ is the direct sum of two ideals, then there is a corresponding decomposition of the manifold $M$ associated with g. Now suppose we start with a $g$ which is indecomposable, i.e., $g$ is not the direct sum of two ideals. We do not know if this already implies that $M$ is weakly irreducible. Note also this phenomenon common to all the $M^{\prime}$ 's so constructed: there is always a distinguished isotropic subspace that is left invariant by the holonomy group. Using the notation at the beginning of the proof of the theorem, this is the subspace $\left\{\left(g^{*},-g^{*}\right): g^{*} \in \mathfrak{g}^{*}\right\}$ of $\vartheta^{-}$.

Proof of Assertions of Example 3. We begin with the simple observation that a connected $G \subseteq P O(V)$ with Lie algebra $g$ leaves a subspace invariant if and only if $\mathfrak{g}$ does, and $G$ acts trivially on a subspace if and only if $g$ annihilates that subspace. We therefore use $\mathrm{g}$ to proves $S-W$ irreducibility since it is much easier to handle $g$ that $G$.

(a) Equip a 3-dimensional $V$ with a scalar product of signature $(2,1)$ and pick an orthonormal basis $\left\{e_{1}, e_{2}, e_{3}\right\}$ of type $(+,+,-)$. Let

$$
\alpha=\left[\begin{array}{ccc}
1 & 1 & 0 \\
-1 & 0 & 1 \\
0 & 1 & 0
\end{array}\right]
$$

relative to $\left\{e_{i}\right\}$, and consider the 1 -dimensional subalgebra $\mathfrak{h}=\operatorname{span}\{\alpha\}$ of $\mathfrak{S}_{\mathfrak{o}}(2,1)$. Exponentiating $\alpha$, we see that the corresponding 1-parameter group $H \subseteq S O(2,1)$ of $\mathfrak{h}$ is in fact the one asserted in the theorem Define a linear map $R: V \wedge V \rightarrow \mathfrak{S D}(2,1)$ by: $R_{e_{1} e_{3}}=0$, 
$R_{e_{1} e_{2}}=R_{e_{2_{2} e_{3}}}=\alpha$. Check that $\{V, R, H\}$ is a riemannian symmetric holonomy system (i.e. verify (1), (2), (6), and (7)) and apply Corollary 2 to obtain a riemannian symmetric space with holonomy group $H$. To see that this space is actually $\boldsymbol{R}^{3}$, observe merely that the Lie algebra $\mathfrak{g}=\mathfrak{h} \oplus V$ (cf. (9)) is solvable so that Proposition 2 applies.

Now clearly $\mathfrak{h}$ annihilates $\left(e_{1}+e_{3}\right)$ and a trivial calculations shows that there is no other 1 -dimensional invariant subspace of $\mathfrak{h}$. As $\left(e_{1}+e_{3}\right)$ is isotropic, $H$ is $S-W$ irreducible, thereby proving $(a)$.

(b) The existence of the manifold is much the same as in part (a). Let $V$ be a 4-dimensional inner-product space of signature $(2,2)$ and let $\left\{e_{1}, \cdots, e_{4}\right\}$ be an orthonormal basis of type $(+,+,-,-)$. Relative to $\left\{e_{i}\right\}$ let $\alpha \in \mathfrak{g} \mathfrak{D}(2,2)$ be:

$$
\alpha=\left[\begin{array}{cccc}
0 & -1 & 0 & 1 \\
1 & 0 & -1 & 0 \\
0 & -1 & 0 & 1 \\
1 & 0 & -1 & 0
\end{array}\right]
$$

and let $\mathfrak{h}=\operatorname{span}\{\alpha\}$. Its corresponding 1 -parameter group in $S O(2,2)$ is therefore the one asserted in the theorem. It remains to define the curvature map $R: V \wedge V \rightarrow \mathfrak{S}(2,2)$ by:

$$
R_{e_{1} e_{3}}=R_{e_{2} e_{4}}=0, R_{e_{1} e_{2}}=R_{e_{3} e_{4}}=R_{e_{2} e_{3}}=-R_{e_{1} e_{4}}=\alpha .
$$

One obtains the desired symmetric space, which is diffeomorphic to $\mathrm{R}^{4}$ because $\mathfrak{g}=\mathfrak{h} \oplus V$ is even nilpotent in this case.

Now define $J: V \rightarrow V$ by: $J e_{1}=e_{2}, J e_{2}=-e_{1}, J e_{3}=e_{4}, J e_{4}=-e_{3}$. Cleary $\alpha \circ J=J \circ \alpha$ and $J^{2}=-I$ ( $I=$ identity). So $R$ is a kahlerian curvature tensor on $V$. We can extend this $J$ to other tangent spaces on $\mathrm{R}^{4}$ either by parallel translation or by left translation by elements of $G$. This complex structure is compatible with the metric, and makes $\mathrm{R}^{4}$ a kahler manifold.

Finally we prove that $H$ is $S-W$ irreducible:

A 1-dimensional subspace of $V$ is left invariant by $\mathfrak{h}$ if and (14) only if it is spanned by an element of the form: $a\left(e_{1}+e_{3}\right)+$ $b\left(e_{2}+e_{4}\right)$ for some $a, b \in \mathbf{R}$.

A 2-dimensional subspace $P$ of $V$ is left invariant by $\mathfrak{h}$ if and only if is either isotropic, or else has a basis $\left\{v_{1}, v_{2}\right\}$ of the form:

$$
\begin{gathered}
v_{1}=a e_{1}+b e_{2}+c e_{3}+d e_{4} \\
v_{2}=(b-d)\left(e_{1}+e_{3}\right)-(a-c)\left(e_{2}+e_{4}\right), a, b, c, d \in \boldsymbol{R} .
\end{gathered}
$$

The "if" parts of both assertions are trivial. Conversely, let $v=a e_{1}$ $+b e_{2}+c e_{3}+d e_{4}$, then 


$$
\alpha(v)=-\left\{(b-d)\left(e_{1}+e_{3}\right)-(a-c)\left(e_{2}+e_{4}\right)\right\} .
$$

Hence if $\mathfrak{h}$ preserves span $\{v\}$, comparison of coefficients gives $a=c$, $b=d$, proving (14). Now, for (15), if $P$ is not isotropic, $\alpha$ does not annihilate every element in it, and so $\left(^{*}\right) \Rightarrow$ there is a $v \in P$ with $a \neq c$ or $b \neq d$. Say $a \neq c$. Then this $v \notin \operatorname{span}\{\alpha(v)\}$, again obvious from $\left.{ }^{*}\right)$. Hence $\{v, \alpha(v)\}$ form a basis of $P$ of the required type. This proves (15).

On the basis of (14) and (15), $S-W$ irreducibility of $H$ is immediate. In fact, (14) implies that all isotropic 1-dimensional subspaces are invariant subspaces, and conversely. By (15), if a 2-dimensional invariant subspace is not isotropic, then it is at least degenerate because, using the notation there, $\left\langle v_{1}, v_{2}\right\rangle=\left\langle v_{2}, v_{2}\right\rangle=0$. This completes the proof of Theorem 3 .

5. We now deal with Theorem 5 and Examples 6, and 4, in this order. These all have to do with the closedness question and for this, we need the following basic result.

Proposition 3. (a) Let $G$ be a connected Lie group and $H$ a connected Lie subgroup of $G$. A necessary and sufficient condition for $H$ to be closed in $G$ is that the closure of every one parameter subgroup of $H$ in $G$ is contained in $H$.

(b) A connected subgroup of the general linear group is closed if and only if its radical is closed in the general linear group.

(c) Let $G$ be a subgroup of the general linear group. Then its commutator subgroup $\mathfrak{D G}$ (which corresponds to $\mathfrak{D g}$ ) is always closed in the general linear group.

Parts (a) and (b) are due jointly to Goto [7] and Malcev [11]. (c) is due to Goto [7], and is an easy consequence of (a) and (b).

Proof of Theorem 5. (a) Let $G \subseteq P O(V)$ be a reductive, weakly irreducible subgroup and assume it is not closed in $P O(V)$. By $(b)$ of Proposition 3, the radical $R$ of $G$ is not closed in $P O(V)$. By (a) of the same proposition, there exists a one-parameter subgroup $h(\alpha)$ of $R$ (with Lie algebra spanned by $\alpha \in g$ ) such that its closure $\overline{h(\alpha)}$ is not in $G$. $\overline{h(\alpha)}$ is then an abelian subgroup and consequently a torus of dimension $\geqq 2$. Let $\bar{\alpha}$ be the Lie algebra of $\overline{h(\alpha)}$. Because $\operatorname{dim} \bar{\alpha} \geqq 2$, there exists an element $\alpha_{1}$ in $\bar{\alpha}$ such that $\alpha_{1}$ annihilates a proper subspace of $V$. Now, $G$ is reductive, so that its radical $R$ coincides with its center. Since $h(\alpha) \subseteq R, \overline{h(\alpha)}$ also commutes with $G$ elementwise. Hence ad $\alpha_{1}$ restricted to $\mathrm{g}$ is identically zero. 
Now, pick an orthonormal basis $\left\{e_{j}\right\}$ of $V$ so that $a_{1}$ assumes the form:

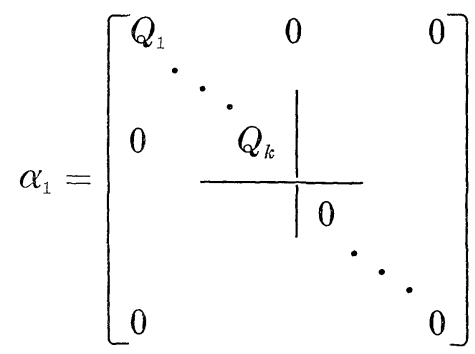

where each $Q_{1}$ is a $2 \times 2$ matrix such that $Q_{i}=\left[\begin{array}{rr}0 & a_{i} \\ -a_{i} & 0\end{array}\right], a_{i} \neq 0$ for $i=1, \cdots, k$. Since $\alpha_{1}$ annihilates a proper subspace of $V, 2 k<d$. In other words, $W=\operatorname{span}\left\{e_{2 k+1}, \cdots, e_{d}\right\}$ is the nontrivial subspace annihilated by $\alpha_{1}$. In this matrix representation of elements of $G$ relative to $\left\{e_{i}\right\}$, let $\mathfrak{p}$ be the natural projection of $\mathfrak{g}$ into those matrices which vanish inside the upper left $(2 k \times 2 k)$-box and the lower right $(d-2 k) \times(d-2 k)$ - box. Take $g \in \mathfrak{g}$, and consider $\left[\alpha_{1}, g\right]=a d\left(\alpha_{1}\right) g$. If $\mathscr{P}(g) \neq 0$, then a trivial computation shows that $\mathscr{P}\left(\left[\alpha_{1}, g\right]\right) \neq 0$ since $a_{i} \neq 0, \forall_{i}=1, \cdots, k$. Thus, a necessary condition for $a d_{\mathfrak{g}} \alpha_{1}=0$ is that: $g \in \mathfrak{g} \Longrightarrow \mathscr{P}(g)=0$. But then $g$ leaves $W$ invariant. Since $W$ is both proper and nondegenerate, this contradicts the weak irreducibility of $G$.

(b) Let $G \leqq P O(V), \operatorname{dim} V \leqq 5$, be weakly irreducible. Assume that it is not closed, then as in part (a), the closure of its radical, $\bar{R}$, must contain a torus of dimension $\geqq 2$. So this already eliminates the cases of dimension of $V$ equal to $1,2,3$. We give below the proof of the case $\operatorname{dim} V=5$; the case of $\operatorname{dim} V=4$ is both similar and simpler.

So $G \leqq P O(V), \operatorname{dim} V=5$, is both weakly irreducible and nonclosed. Let $\bar{\alpha}$ be the Lie algebra of the 2-dimensional torus contained in $\bar{R}$. We may assume that relative to a well chosen orthonormal basis $\left\{e_{\imath}\right\}, \bar{\alpha}$ is exactly the linear span of:
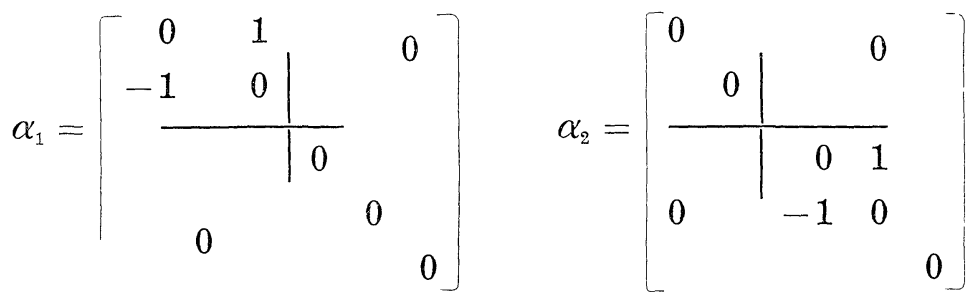

Denote the algebras of $R, \bar{R}, \bar{G}$ by $\mathfrak{x}, \overline{\mathfrak{x}}, \overline{\mathfrak{g}}$. Then $\alpha_{1}, \alpha_{2} \in \overline{\mathfrak{x}}$ and we claim: $\alpha d \alpha_{1}, \alpha d \alpha_{2}$ map $\overline{\mathfrak{g}}$ into $\overline{\mathfrak{x}}$.

For, $R$ is the radical of $G$, which is invariant in $\bar{G}$ as is well-known; 
so $R$ is invariant in $\bar{G}$ because the radical is a characteristic subgroup. But closures of invariant subgroups remain invariant, hence $\bar{R}$ is invariant in $\bar{G}$. Then $[\overline{\mathfrak{x}}, \bar{g}] \leqq \overline{\mathfrak{x}}$, which implies (16).

Relative to $\left\{e_{i}\right\}$, let $\mathscr{P}$ be the natural projection of $\overline{\mathrm{g}}$ into those matrices which vanish outside of the following mutually symmetric boxes: that consisting of the $(1,3)-,(1,4)-,(2,3)-,(2,4)$-th entries and that consisting of the $(3,1)-,(4,1)-,(3,2)-,(4,2)$-th entries. A trivial computation shows that $g \in \mathfrak{g} \Rightarrow \mathscr{P}(g)=\left(a d \alpha_{2}\right)^{2} \circ\left(a d \alpha_{1}\right)^{2}(g)$. So by (16), we get:

$$
g \in \mathfrak{g} \Rightarrow \mathscr{P}(g) \in \overline{\mathfrak{g}} .
$$

Now, if $g=\left\{g_{i j}\right\}$ relative to $\left\{e_{i}\right\}$, then $g^{\prime} \equiv\left(g-g_{12} \alpha_{1}-g_{34} \alpha_{2}-\mathscr{P}(g)\right)$ is a matrix whose entries are all zero except that $\left(g_{15}^{\prime}, g_{25}^{\prime}, g_{35}^{\prime}, g_{45}^{\prime}\right)=\left(g_{15}\right.$, $\left.g_{25}, g_{35}, g_{45}\right)$. By (17) $g^{\prime} \in \overline{\mathfrak{g}}$. If there is a $g \in \mathfrak{g}$ such that $\left(g_{15}, \cdots, g_{45}\right) \neq 0$, then there is a $g^{\prime} \in \bar{g}$ such that each entry of $g^{\prime}$ is zero except that $\left(g_{15}^{\prime}, \cdots, g_{45}^{\prime}\right)=\left(g_{15}, \cdots, g_{45}\right) \neq 0$. Specifically, let $\left(g_{15}, g_{25}\right) \neq 0$. Then $-\left(\alpha d \alpha_{1}\right)^{2}\left(g^{\prime}\right) \equiv g^{\prime \prime}$ is a matrix whose only nonzero entries are the $(1,5)-$, $(2,5)$-th ones, where they are equal to $\left(g_{15}, g_{25}\right)$ respectively. Thus $\left(g^{\prime \prime}\right)^{2}\left(e_{5}\right)= \pm\left(g_{15}^{2}+g_{25}^{2}\right) e_{5}$, where the sign depends on whether $\left\langle e_{5}, e_{5}\right\rangle$ $=\left\langle e_{1}, e_{1}\right\rangle$ or $-\left\langle e_{1}, e_{1}\right\rangle$. In any case, $\left(g_{15}, g_{25}\right) \neq 0 \Rightarrow g^{\prime \prime}$ is not nilpotent.

Now, by definition, $g^{\prime \prime} \in[\overline{\mathfrak{g}}, \overline{\mathrm{x}}]$. Since $[\overline{\mathfrak{x}}, \overline{\mathfrak{x}}] \subseteq \overline{\mathfrak{x}}$, and we have shown, that $\overline{\mathfrak{x}}$ is an ideal of $\overline{\mathfrak{g}}, \overline{\mathrm{x}}$ is therefore a solvable ideal of $\overline{\mathfrak{g}}$ and consequently contained in the radical $\mathfrak{r}^{\prime}$ of $\overline{\mathrm{g}}$. (Actually $\mathfrak{q}^{\prime}=\overline{\mathrm{r}}$.) Thus $g^{\prime \prime} \in\left[\mathfrak{g}, \mathfrak{x}^{\prime}\right]$ which is the nilpotent radical of $\bar{g}$. Hence $g^{\prime \prime}$ has to be a nilpotent matrix, which contradicts what we proved above.

Thus $g \in \mathfrak{g} \Rightarrow\left(g_{15}, g_{25}\right)=0$. Exactly the same argument shows that necessarily also $\left(g_{35}, g_{45}\right)=0$. But this means $g$ annihilates $e_{5}$, and $G$ cannot be weakly irreducible. Then $G$ was closed in $P O(V)$ to start with.

(c) We preface the proof by a general remark on the complexification $V^{c}$ of a real vector space $V$. We denote the complex general linear group by $G l(V, C)$, with Lie algebra $\operatorname{gl}(V, C)$ Let $\alpha \in \mathfrak{g l}(V)$ and suppose that its corresponding 1-parameter subgroup $h(\alpha) \subseteq G l(V)$ has compact closure. Then it is well-known that $\alpha$ is conjugate in $G(V, C)$ to a diagonal matrix whose entries are pure imaginary or zero. In other words, there exists a basis of $V^{c}$ relative to which $\alpha$ is diagonal with pure imaginary or zero entries. Conversely, if $\alpha \in \mathfrak{g l}(V)$ has the property that its eigenvalues are either pure imaginary or zero and that it is diagonalizable, then $h(\alpha)$ has compact closure. Hence we have:

Given $\alpha \in \operatorname{gl}(V)$, then $h(\alpha)$ is relatively compact in $G(V)$ if and only if there exists a basis $\left\{v_{\imath}\right\}$ of $V^{\circ}$ over $C$ such that $\alpha\left(v_{i}\right)=\nu_{i} v_{i}$, where $\nu_{i}$ is either pure imaginary or zero, $i=$ $1, \cdots, d$.

We now turn to the proof of the theorem. Let $M$ be a given Sym- 
metric space. Since we are only interested in the identity component of the holonomy group, we may as well assume $M$ to be simply connected to start with. Corollary 3 of Section 3 applies, and we let $M$ be expressed in "reduced form" $G / K$, i.e., there is a group involution $\sigma: G \rightarrow G$ such that $K$ is the identity component of the fixed point set of $\sigma$ and

$$
\mathfrak{g}=\mathfrak{t} \oplus \mathfrak{p} ;[\mathfrak{k}, \mathfrak{k}] \subseteq \mathfrak{f},[\mathfrak{f}, \mathfrak{p}] \subseteq \mathfrak{p},[\mathfrak{p}, \mathfrak{p}]=\mathfrak{f} .
$$

Here $\mathfrak{p}$ is the $(-1)$-eigenspace of $(d \sigma)_{e}$. The holonomy group of $M$ is $A d_{\mathfrak{p}}(K)$.

Consider the adjoint representation of $G$ in $\mathfrak{g}, A d(G) \subseteq G \mathrm{l}(\mathrm{g})$. The commutator subgroup $\mathscr{D}(A d(G))=A d(\mathscr{D} G)$ is closed in $G \mathrm{I}(\mathfrak{g})$, by Proposition 3 (c). The algebra of $\mathscr{D} G$ is $\mathscr{D} \mathfrak{g}=\mathfrak{l} \oplus[\mathfrak{f}, \mathfrak{p}]$, by (19). Hence $K \subseteq \mathscr{D} G$. Furthermore $\sigma$ induces an involution in $\mathscr{D} G$ which can be carried to $A d(\mathscr{D} G)$ as follows: $A d$ induces an isomorphism of $\mathscr{D} G / Z$ onto $A d(\mathscr{D} G)$, where $Z$ is the center of $\mathscr{D} G$. The involution on $\mathscr{D} G$ clearly maps $Z$ onto $Z$ itself so that the involution on $\mathscr{D} G / Z$ is well-defined. We carry it to $A d(\mathscr{D} G)$ via the above isomorphism and call it $\hat{\sigma}$. We claim that the identity component of the fixed point set of $\widetilde{\sigma}$ is precisely $A d(K)$. For this, one notes that in $\mathscr{D} G$, the fixed point set of the involution has $K$ as its identity component, because $\mathscr{D} \mathfrak{g}=\mathfrak{H} \oplus[\mathfrak{f}, \mathfrak{p}]$. Then, in $\mathscr{D} G / Z$, the algebra of the fixed point set is $\mathfrak{t} / \mathfrak{t} \cap z$ (where $z$ is the algebra of $Z$ ), and so the corresponding connected subgroup is $K Z / Z$. But then $A d$ maps $K Z / Z$ isomorphically onto $A d(K)$, hence our claim. Thus, $A d(K)$ is closed in $A d(\mathscr{D} G)$ which is closed in $G \mathfrak{l}(\mathrm{g})$. Therefore, $A d(K)$ is closed in $G l(\mathrm{~g})$.

Now, by (19), $A d(K) \subseteq A d_{\mathfrak{k}}(K) \times A d_{\mathfrak{p}}(K)$. We are interested in showing that the projection of $A d(K)$ onto its second factor is closed. For this, we need a lemma for the proof of which I am much indebted to Professor Borel.

LEMMA. Let a Lie algebra $g$ admit a decomposition satisfying (19). Suppose $\alpha \in \mathfrak{f}$ such that the one-parameter group corresponding to $a d_{\mathfrak{p}}(\alpha)$ is relatively compact in $G \mathrm{I}(\mathfrak{p})$. Then the one-parameter group corresponding to ad $(\alpha)$ in $\mathrm{GI}(\mathrm{g})$ is also relatively compact.

Proof. By (18), we may assume a $C$-basis $\left\{f_{i}\right\}_{i \leqq i \leqq d}$ so chosen in $\mathfrak{p}^{c}$ that

$$
\left(a d_{\mathfrak{p}} \alpha\right)\left(f_{i}\right)=\mu_{i} f_{i}, \quad i=1, \cdots, d,
$$

where each $\mu_{i}$ is either pure imaginary or zero. Since $[\mathfrak{p}, \mathfrak{p}]=\mathfrak{l}$, $\left[\mathfrak{p}^{c}, \mathfrak{p}^{c}\right]=\mathfrak{l}^{c}$. So $\left\{\left[f_{i}, f_{j}\right]\right\}_{1 \leq i, j \leqq d}$ span $\mathfrak{f}^{c}$. We subtract from the latter a $\mathbb{C}$-basis $\left\{\left[f_{\alpha}, f_{\beta}\right]\right\}_{(\alpha \beta \beta) \in A \times B}$ for $\mathfrak{f}^{c}$. Define $k_{\alpha \beta}=\left[f_{\alpha}, f_{\beta}\right]$. Then we have: 


$$
\begin{aligned}
\left(\alpha d_{\mathfrak{f}} \alpha\right)\left(k_{\alpha \beta}\right) & =\left[\alpha,\left[f_{\alpha}, f_{\beta}\right]\right] \\
& =-\left[f_{\alpha},\left[f_{\beta}, \alpha\right]\right]-\left[f_{\beta},\left[\alpha, f_{\alpha}\right]\right] \\
& =\left[f_{\alpha},\left(a d_{\mathfrak{p}} \alpha\right) f_{\beta}\right]+\left[\left(a d_{\mathfrak{p}} \alpha\right) f_{\alpha}, f_{\beta}\right] \\
& =\left(\mu_{\alpha}+\iota_{\beta}\right)\left[f_{\alpha}, f_{\beta}\right] \\
& \equiv \lambda_{\alpha \beta} k_{\alpha \beta} .
\end{aligned}
$$

Clearly, $\lambda_{\alpha \beta}$ so defined are all either pure imaginary or zero. So relative to the $\boldsymbol{C}$-basis $\left\{k_{\alpha \beta}, f_{i}\right\}_{(\alpha, \beta) \in A \times B, 1 \leqq i \leqq d}$ of $\mathrm{g}^{\mathrm{c}},(a d \alpha)$ is diagonal with either zero or pure imaginary entries. The lemma now follows from (18).

Returning to the proof of the theorem, suppose $A d_{\mathfrak{p}}(K)$ is not closed in $G(\mathfrak{l})$. By Proposition 3 (a) as well as the usual argument, there is an $\alpha \in \mathfrak{f}$ such that the one-parameter subgroup $h(\alpha)$ corresponding to $\left(a d_{\mathfrak{p}} \alpha\right)$ has compact closure $\overline{h(\alpha)} . \overline{h(\alpha)}$ is in $G \mathfrak{l}(\mathfrak{p})$ but not in $A d_{\mathfrak{p}}(K)$. By the lemma, the one parameter group $h^{\prime}(\alpha)$ corresponding to $\left(a d_{p} \alpha\right)$ also has compact closure in $\operatorname{GI}(g)$. So for a suitable choice of basis $\left\{e_{\jmath}\right\}$ in $\mathfrak{g}$, we know that the matrix of $(a d \alpha)$ has the form:

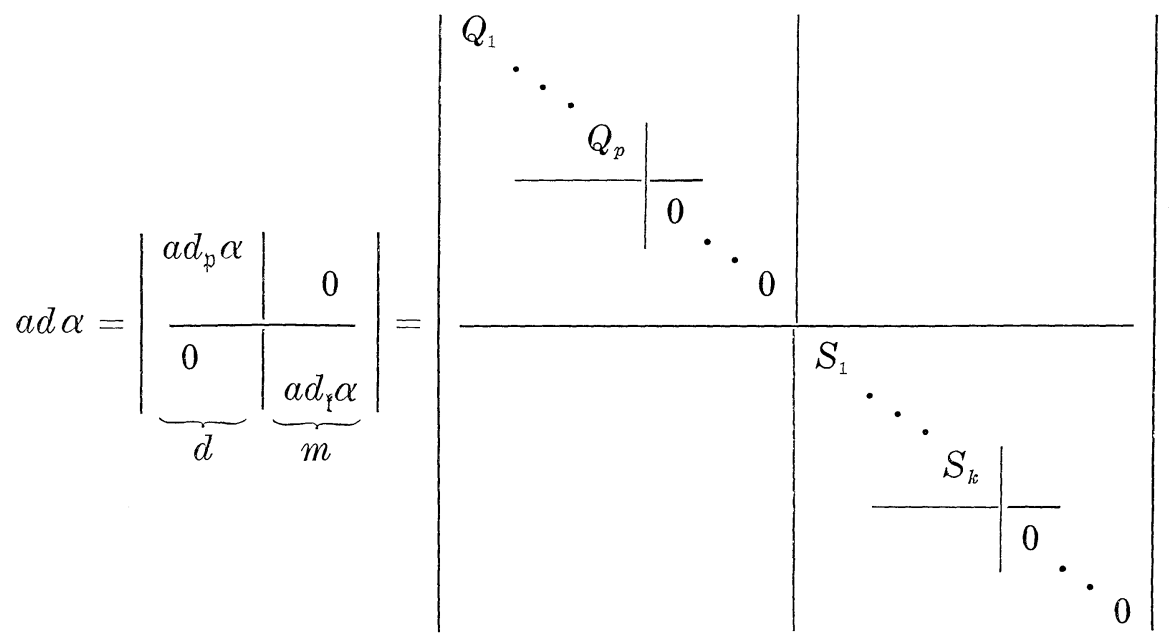

where

$$
Q_{i}=\left[\begin{array}{rr}
0 & \lambda_{i} \\
-\lambda_{i} & 0
\end{array}\right] \text { and } S_{j}=\left[\begin{array}{cc}
0 & \mu_{j} \\
-\mu_{j} & 0
\end{array}\right]
$$

are $2 \times 2$ matrices such that $\lambda_{i} \neq 0$ for all $1 \leqq i \leqq p, \mu_{j} \neq 0$ for all $1 \leqq j \leqq k$. Since $\overline{h(\alpha)} \leqq A d_{\mathfrak{p}}(K)$, we may assume that there is some $A$ such that $\exp A$ is not in $A d_{\mathfrak{p}}(K)$, where $A$ is the $d \times d$ matrix: 


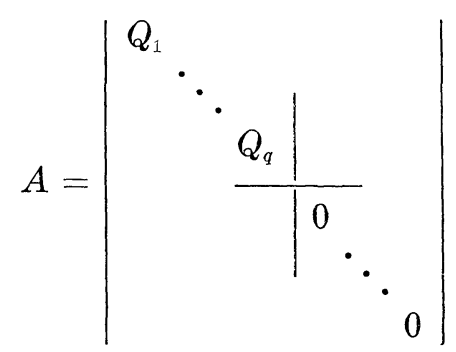

and $q<p$. Let $\left\{\mu_{i_{1}}, \cdots, \mu_{i_{1}}\right\}$ be the set of all $\mu_{j}^{\prime}$ s commensurable with $\left.\lambda_{1}, \cdots \lambda_{q}\right\}$; for convenience, let these be $\left\{\mu_{1}, \cdots, \mu_{\mathfrak{l}}\right\}$, where $\mathfrak{l} \leqq k$, i.e., $\lambda_{1}, \cdots, \lambda_{q}, \mu_{1}, \cdots, \mu_{\mathfrak{r}}$ are linearly dependent over the integers. We claim that if $B$ is the following $(d+m) \times(d+m)$-matrix:

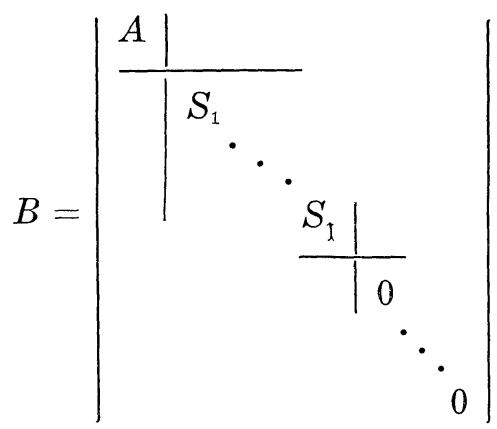

then $\exp B$ cannot be in $A d(K)$. For if it were, $a d_{\mathfrak{p}} B=A$ and $A$ will be in $\alpha d_{\mathfrak{p}}(\mathfrak{f})$, which contradicts $\exp A \notin A d_{\mathfrak{p}}(K)$. But from the form of $a d \alpha$, the standard arguments show that $\exp B$ is a torus contained in $\overline{h^{\prime}(\alpha)}$, which is itself a torus in $G(\mathrm{~g})$. Hence $\overline{h^{\prime}(\alpha)} \nsubseteq A d(K)$, and this shows that $A d(K)$ is not closed in $G((\mathrm{~g})$. This flatly contradicts the fact established above, that $A d(K)$ is closed in $G(\mathfrak{g})$. So, $A d_{\mathfrak{p}}(K)$ had better be closed in $G r(p)$.

Proof of Assertions of Example 6. Take an inner product space of dimension 6 with signature $(4,2)$ and let $\left\{e_{i}\right\}$ be an orthonormal basis of type $(+,+,+,+,-,-)$. Let $\mathfrak{h}^{*}=\operatorname{span}\{\alpha, \beta, \gamma, \delta\}$ be the subspace of $\mathfrak{s}(4,2)$ such that relative to $\left\{e_{i}\right\}$ :

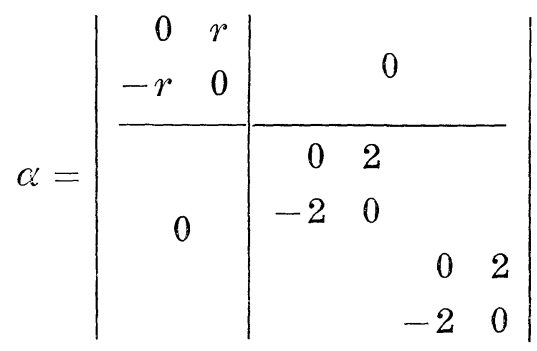

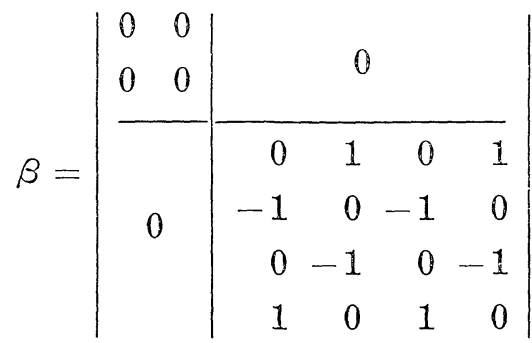




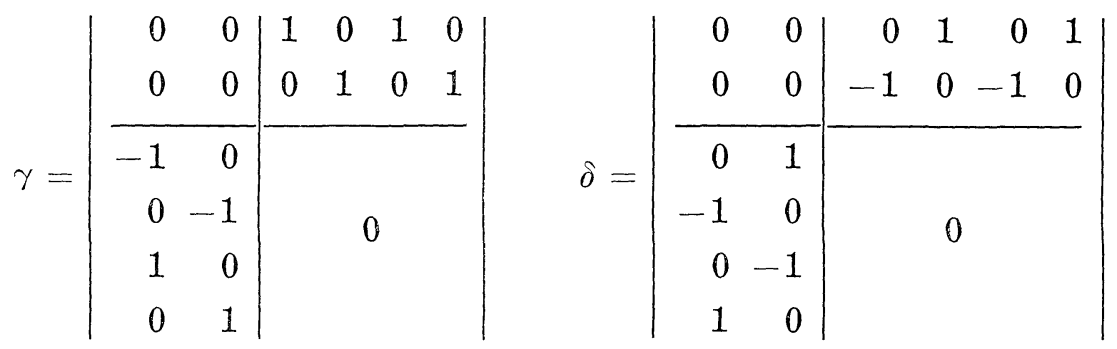

Here $r \in \boldsymbol{R}$ is arbitrary for the moment. Note that since: $[\alpha, \gamma]=$ $(\gamma-2) \delta,[\alpha, \delta]=-(r-2) \gamma,[\gamma, \delta]=-2 \beta$, and all other brackets are zero, $\mathfrak{h}^{*}$ is in fact a solvable subalgebra of $\mathfrak{g}(4,2)$. We denote as usual its corresponding connected subgroup by $H^{*}$. Now, define $R: V \wedge V \rightarrow \mathfrak{h}^{*} \subseteq \mathfrak{o}(4,2)$ by:

$$
\begin{aligned}
& R_{e_{1} e_{2}}=r \beta \\
& R_{e_{1} e_{3}}=R_{e_{1} e_{5}}=R_{e_{e^{e_{4}}}}=R_{e_{2^{e_{6}}}}=(r / 2) \gamma \\
& R_{e_{1} e_{4}}=R_{e_{1} e_{6}}=-R_{e_{2} e_{3}}=-R_{e_{2} e_{5}}=(r / 2) \delta \\
& R_{e_{3} e_{6}}=-R_{e_{4} e_{5}}=\alpha \\
& R_{e_{3} e_{5}}=R_{e_{4} e_{6}}=0 \\
& R_{e_{3 e_{4}}}=\alpha+2 \beta \\
& R_{e_{5} e_{6}}=\alpha-2 \beta \text {. }
\end{aligned}
$$

We claim that this is a kahlerian curvature tensor, i.e., one has to verify (1)-(3). (2) is true by definition. For the proof (1), one goes through 20 such identities, and we leave that out. For (3), define $J: V \rightarrow V$ by $J e_{2 i+1}=e_{2 i+2}, J e_{2 i+2}=-e_{2 i+1}, i=0,1,2$. It is then clear that $J^{2}=-\mathrm{I}$ and $J \circ R_{x y}=R_{x y} \circ J$ for all $x, y \in V$. Therefore $H^{*}$ is an algebraic kahlerian holonomy group.

So far, $r$ is arbitrary. If we let it take on irrational values, then $\alpha$ corresponds to a Kronecker line dense in a 2-dimensional torus, which cannot be in $H^{*}$. Proposition 3 (a) tells us that $H^{*}$ is not closed in $S O(4,2)$ in this case.

REMARK 6. Theorem 5 (c) tells us that for irrational $r,\left\{V, R, H^{*}\right\}$ cannot be a symmetric holonomy system, in view of Corollary 2 of Section 3. However, it may be interesting to point out that when (and only when) $r=1,\left\{V, R, H^{*}\right\}$ is indeed symmetric, and we have therefore a concrete example of a hermitian symmetric space whose holonomy group is solvable but not abelian.

Proof of Assertions of Example 4. We will prove the theorem in this order: construct the algebra $g$ of the group $G$, show that $G$ cannot be the holonomy group of a riemannian manifold, and finally prove that $G$ is $S-W$ irreducible. The bulk of the proof lies in demonst- 
rating $S-W$ irreducibility.

Let $V$ be an inner product space of signature $(5,1)$, and $\left\{e_{i}\right\}$ is an orthonormal basis of type $(+,+,+,+,+,-)$. Consider the subspace $\mathfrak{g}$ of $\mathfrak{g}(5,1)$ spanned by the following matrices (relative to $\left.\left\{e_{i}\right\}\right)$ :

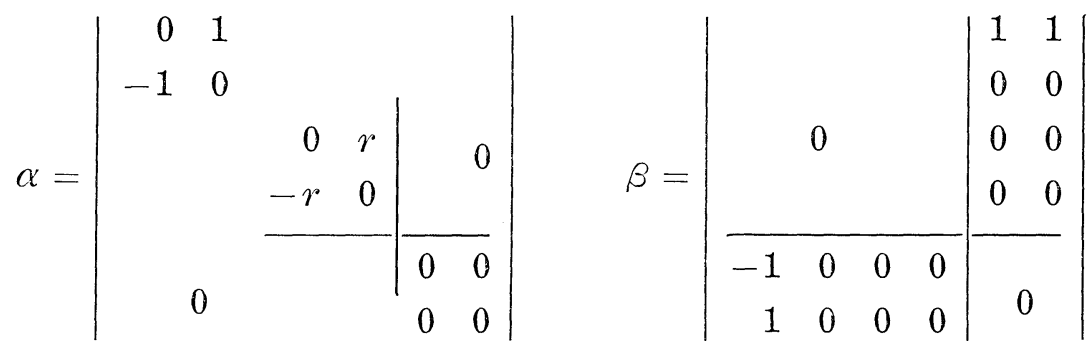

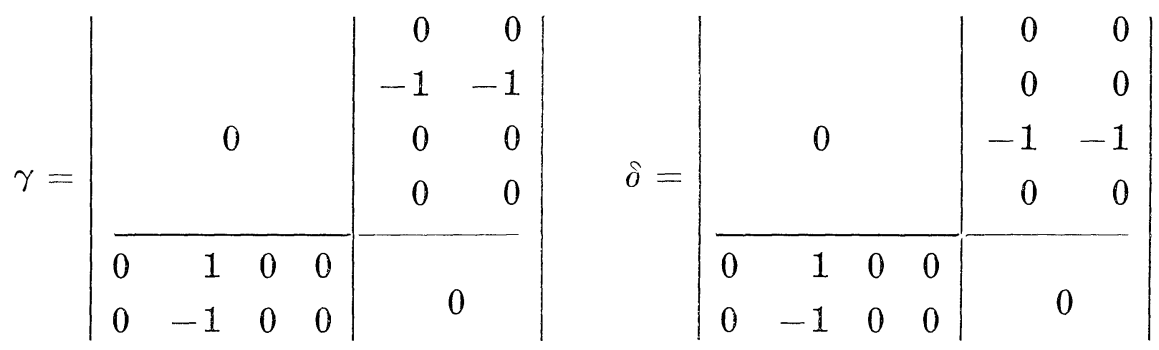

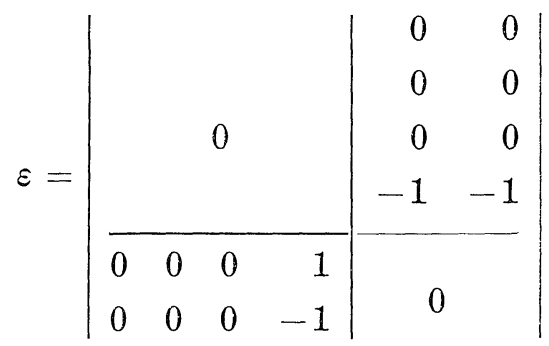

Again we let $r \in \boldsymbol{R}$ be arbitrary for the moment. Clearly $[\alpha, \beta]=\gamma$, $[\alpha, \gamma]=-\beta,[\alpha, \delta]=r \varepsilon,[\alpha, \varepsilon]=-r \delta$, and all other brackets are zero. Hence $g$ is a subalgebra and let $G \subseteq S O(5,1)$ be its corresponding subgroup.

Now, suppose $r$ is irrational. By the same argument used in the proof of Theorem 6, $G$ is not closed in $G I(V)$. Suppose $r$ is rational, then we claim $G$ is closed. For the only element of $g$ tangent to the maximal compact subgroup $S O(5)$ of $S O(5,1)$ is $\alpha$, which is easy to see. But $\alpha$ corresponds to a circle in this case. Hence the closures of all one-parameter subgroups of $G$ are in $G$, which implies $G$ is closed by virtue of Proposition 3 (a).

We have therefore found both closed and nonclosed subgroups by varying $r$. It will be convenient for later purposes if we restrict ourselves to the case:

$$
r>1
$$


regardless of whether $r$ is rational or not. The following arguments apply so long as (20) holds. So, we shall be proving the theorem simultaneously for both closed and nonclosed $G$ 's. First, we shall show that $G$ cannot even be an algebraic riemannian holonomy group. By Remark 1 of Section 2, a fortiori, it cannot be a genuine holonomy group.

Suppose it is, there exist then riemannian curvature tensors $R^{1}, \cdots, R^{s}$ on $V$ such that:

$$
\operatorname{span}\left\{R_{e_{i} e_{j}}^{k}\right\}_{1 \leqq i<j \leqq 6}=\mathfrak{g}, \quad k=1, \cdots, s .
$$

Let $R$ be any one of the $R^{k}$ and we claim:

$$
\operatorname{span}\left\{R_{\left.e^{\ell e_{j}}\right\}_{1<j, \mathfrak{f}=12} \subseteq} \leqq \operatorname{span}\{\beta, \gamma, \delta, \varepsilon\} .\right.
$$

Suppose (22) were false. Let $\mathfrak{l}=1$. So there exists $0 \neq t \in \boldsymbol{R}$ such that $R_{e_{1} e_{3}}=t \alpha+\{\beta, \cdots, \varepsilon\}$. Here, $\{\beta, \cdots, \varepsilon\}$ stands for some linear combination of $\beta, \gamma, \delta, \varepsilon$. According to (1),

$$
R_{e_{1} e_{j}} e_{3}+R_{e_{j} e_{3}} e_{1}+R_{e_{3} e_{1}} e_{j}=0 \text {. }
$$

By above,

$$
\{t \alpha+\{\beta, \cdots, \varepsilon\}\} e_{3}+R_{e^{e_{3}} e_{1}} e_{1}+R_{e_{e^{e}} e_{j}} e_{j}=0,
$$

and $t \neq 0$. Now, all $(1,3)$-th entries of elements of $g$ are zero, and so (4) of Section 2 implies that $R_{e_{1} e_{3}}=0$. So the above is equivalent to $\{t \alpha+\{\beta, \cdots, \varepsilon\}\} e_{3}+R_{e_{1 e_{3}}} e_{1}=0$. But $\mathrm{g}$ maps $e_{1}$ into the span of $\left\{e_{2}, e_{5}, e_{6}\right\}$, and $\beta, \gamma, \delta, \varepsilon$ all move $e_{3}$ into the span of $\left\{e_{5} e_{6}\right\}$. Hence if we take the inner product of the above equation with $e_{4}$, we get $\left\langle(t \alpha) e_{3}, e_{4}\right\rangle=0$; i.e., $-t r=0, \Rightarrow t=0$ by $(20)$. This contradiction proves (22) for $\mathfrak{l}=1$. The proof for $\mathfrak{l}=2$ is exactly the same.

In a similar fashion, one has:

$$
\operatorname{span}\left\{R_{e e_{e}}\right\}_{\{<j,\{=3,4} \subseteq \operatorname{span}\{\beta, \gamma, \delta, \varepsilon\} .
$$

The proof of this differs from that of (22) only in using

$$
R_{e_{\ell e j}} e_{1}+R_{e_{j} e_{1}} e_{\mathfrak{I}}+R_{e_{1} e_{\ell}} e_{j}=0
$$

and at the end take inner product with $e_{2}$. So combining (22) and $(22)^{\prime}$, we have:

$\operatorname{span}\left\{R_{e_{i} e_{j}}^{k}\right\}_{i<j} \subseteq \operatorname{span}\{\beta, \gamma, \delta, \varepsilon\}$ for $i=1,2,3,4, k=1, \cdots, s$. But since the $(5,6)$-th entry of every element of $g$ is zero, (4) implies $R_{e_{5} e_{6}}=0$. Together, these imply

$$
\operatorname{span}\left\{R_{e_{i} e_{j}}^{k}\right\}_{1 \leqq i<j \leqq 6} \subseteq \operatorname{span}\{\beta, \gamma, \delta, \varepsilon\} \text { for } k=1, \cdots, s \text {. }
$$

This contradicts (21). Hence we have proved that $g$ is not the algebra of an algebraic riemannian holonomy group. 
Finally, we turn to the proof of $S-W$ irreducibility of $G$, for which we must determine $A L L$ invariant subspaces of $g$. The proof is fairly tricky, even if elementary, so we present it in detail.

(23) The only invariant 1 -dimensional subspace of $G$ is $\operatorname{span}\left\{e_{5}-e_{6}\right\}$.

Proof of (23). Let $\operatorname{span}\{u\}$ be preserved by $g$ and let

$$
u=\left(u_{1}, \cdots, u_{6}\right) \equiv \sum_{i} u_{i} e_{i} .
$$

By definition,

$$
\alpha(u)=\left(u_{2},-u_{1}, r u_{4},-r u_{3}, 0,0\right) .
$$

Thus $\alpha(u) \in \operatorname{span}\{u\} \Rightarrow u_{1}=u_{2}=u_{3}=u_{4}=0$. Hence $u=u_{5} e_{5}+u_{6} e_{6}$. Now, $\beta(u)=\left(u_{5}=u_{6}\right) e_{1} \in \operatorname{span}\{u\} \Rightarrow u_{5}+u_{6}=0, \Rightarrow u=u_{5}\left(e_{5}-e_{6}\right), u_{5}$ arbitrary. Conversely, span $\left(e_{5}-e_{6}\right\}$ is clearly preserved by $\mathfrak{g}$.

There are no invariant 2-dimensional subspaces.

Proof of (24). Let $P=\operatorname{span}\{u, v\}$ be a 2-dimensional invariant space, and we will show this is impossible. As usual, $u=\left(u_{1}, \cdots, u_{6}\right)$, $v=\left(v_{1}, \cdots, v_{6}\right)$. We show first:

$$
\alpha(u) \notin \operatorname{span}\{u\} \text {. }
$$

For, suppose the contrary, then $u=u_{5} e_{5}+u_{6} e_{6}$. But

$$
\alpha(v) \in P \Rightarrow \alpha(v)=a v+b u
$$

for some $a, b \in R, \Rightarrow\left(a v_{1}, a v_{2}, a v_{3}, a v_{4}, a v_{5}+b u_{5}, a v_{6}+b u_{6}\right)\left(v_{2},-v_{1}, r v_{4}\right.$, $\left.-r v_{3}, 0,0\right)$. Equality of the first four terms implies

$$
v_{1}=v_{2}=v_{3}=v_{4}=0 \text {. }
$$

So $v=v_{5} e_{5}+v_{6} e_{6}$. Since also $\beta(v) \in P, \Longrightarrow v_{5}=-v_{6}$, and

$$
\beta(u) \in P \Longrightarrow u_{5}=-u_{6} .
$$

Altogether, they imply that $u, v$ are dependent

So by $\left(^{*}\right)$ we may assume that $P=\operatorname{span}\{u, \alpha(u)\}$. Now

$$
\alpha^{2}(u) \in P \Rightarrow \alpha^{2}(u)=a u+b \alpha(u)
$$

for some $a, b \in \boldsymbol{R}, \Longrightarrow\left(-u_{1},-u_{2},-r^{2} u_{3},-r^{2} u_{4}, 0,0\right)=\left(a u_{1}+b u_{2}, a u_{2}-\right.$ $\left.b u_{1}, a u_{3}+b r u_{1}, a u_{\ddagger}-b r u_{3}, a u_{5}, b u_{6}\right)$. If $a=0$, we may assume $b \neq 0$ since otherwise

$$
\alpha^{2}(u)=0, \Rightarrow u_{1}=u_{2}=u_{3}=u_{4}=0, \Rightarrow \alpha(u) \in \operatorname{span}\{u\},
$$

contradicting $\left({ }^{*}\right)$. Hence in the event that $a=0, b \neq 0$. Consequently, 
equality of the first two terms above $\Rightarrow-u_{1}=b u_{2}=r^{2} u_{1} \Rightarrow u_{1}=0$ by $(20), \Rightarrow$ by virtue of the equality of the first terms that $u_{2}=0$. Similarly, equality of the third and fourth terms

$$
\Rightarrow-r^{2} u_{3}=b r u_{4}=b^{2} u_{3} \Rightarrow u_{3}=0 \Rightarrow u_{4}=0 .
$$

So again, $\alpha(u) \in \operatorname{span}\{u\}$.

We have therefore proved that if $\alpha^{2}(u)=a u+b \alpha(u)$, then $a \neq 0$, Then equality of the coefficients of

$$
e_{5}, e_{6} \Rightarrow u_{5}=u_{6}=0 \Longrightarrow u=\left(u_{1}, u_{2}, u_{3}, u_{4} 0,0\right) .
$$

But $\beta(u)=\left(0, \cdots, 0,-u_{1}, u_{2}\right)=c u+d \alpha(u)$ for some

$$
c, d \in \boldsymbol{R} \Rightarrow u_{1}=u_{2}=0 .
$$

Applying $\delta$ to $u$ gives similarly $u_{3}=u_{4}=0$, and this again contradicts $u$ being a basis element of $P$. (24) is thereby proved.

A 3-dimensional subspace is invariant if and only if it has a basis $\{u, v, w\}$ of the form:

$$
\begin{aligned}
u & =\left(u_{1}, u_{2}, u_{3}, u_{3}, A,-A\right) \\
v & =\left(u_{2},-u_{1}, r u_{4},-r u_{3}, 0,0\right) \\
w & =(0, \cdots, 0, B,-B)
\end{aligned}
$$

where $u_{1}, \cdots, u_{4}, A, B \in \boldsymbol{R}$ are arbitrary.

Proof of (25). Let $P$ be a 3-dimensional invariant subspace. Since the maximal subspace on which $\alpha$ leaves invariant every 1-dimensional subspace is $\operatorname{span}\left\{e_{5}, e_{6}\right\}$-which is only 2-dimensional-we can certainly find a $u \in P$ such that $\alpha(u) \notin \operatorname{span}\{u\}$. Hence if $u=\left(u_{1}, \cdots, u_{6}\right), u_{1} \neq 0$ for some $i \in\{1,2,3,4\}$. For the sake of definiteness, let $i=1$ for the moment. So $u=\left(u_{1}, \cdots, u_{6}\right), u_{1} \neq 0$, and

$$
v=\alpha(u)=\left(u_{2},-u_{1}, r u_{4},-r u_{3} 0,0\right)
$$

are lineary independent elements of $P$. Consider

$$
w \equiv \beta(u)=\left(u_{5}+u_{6}, 0,0,0,-u_{1}, u_{1}\right) \in P .
$$

We claim that $\{u, v, w\}$ are linearly independent. If not, there would exist $a, b \in \boldsymbol{R}$ such that $w=a u+b v$. In other words:

$$
\begin{aligned}
u_{5}+u_{6} & =a u_{1}+b u_{2} ; 0=a u_{2}-b u_{1} ; 0=a u_{3}+b r u_{4} ; \\
0 & =a u_{4}-b r u_{3} ;-u_{1}=a u_{5} ; u_{1}=a u_{6} .
\end{aligned}
$$

The last two equations imply $a \neq 0$, and 


$$
u_{5}+u_{6}=0 \Rightarrow b \neq 0, u_{2} \neq 0
$$

by virtue of the first equation. So the first two equations imply

$$
\begin{aligned}
a u_{1}+b u_{2} & =0, a u_{2}-b u_{1}=0, \Rightarrow a^{2} u_{1}=-b\left(a u_{2}\right)=-b\left(b u_{1}\right) \\
& =-b^{2} u_{1}, \Rightarrow a^{2}=-b^{2},
\end{aligned}
$$

which cannot be. Thus $\{u, v, w\}$ is a basis for $P$. We now show that necessarily $u_{5}+u_{6}=0$. For $\gamma(v) \in P \Rightarrow$ there exist $m, n, \mathfrak{l} \in \boldsymbol{R}$ such that $\gamma(v)=m u+n v+\mathfrak{l} w$. Writing things out term by term as before, we have:

$$
\begin{aligned}
& 0=m u_{1}+n v_{2}+\mathfrak{l}\left(u_{5}+u_{6}\right) ; 0=m u_{2}-n u_{1} ; 0=m u_{3}+n r u_{4} ; \\
& 0=m u_{4}-n r u_{3} ; u_{1}=m u_{5}-\mathfrak{l} u_{1} ;-u_{1}=m u_{6}+\mathfrak{l} u_{1} .
\end{aligned}
$$

If $u_{5}+u_{6} \neq 0$, the last two equations would imply $m=0$, $\Rightarrow$ by virtue of the second equation that $n=0, \Rightarrow \mathfrak{l}=0$ by virtue of the first, $\Rightarrow \gamma(v)=0$. But this contradicts $u_{1} \neq 0$ since $\gamma(v)=\left(0, \cdots, 0, u_{1},-u_{1}\right)$. Thus, $u_{5}+u_{6}=0$ and consequently:

$$
\begin{aligned}
u & =\left(u_{1}, u_{2}, u_{3}, u_{4}, u_{5}\right), u_{1} \neq 0 \\
v & \left.=u_{2},-v_{1}, r u_{4},-r u_{3}, 0,0\right) \\
w & \left.=0, \cdots, 0,-u, u_{1}\right)
\end{aligned}
$$

constitute a basis of the desired form. Now, if we had assumed earlier that $u_{2} \neq 0, u_{3} \neq 0, u_{s} \neq 0$ respectively, we would then have let $w=\gamma(u), \delta(u), \varepsilon(u)$ resp., and would have applied $\beta, \delta, \varepsilon$, to $v$ resp. to get also the fact that $u_{5}+u_{6}=0$. We would still have obtained a basis of the form above. This proves completely the existence of the form above. This proves completely the existence of the basis as claimed. Conversely, it is trivial that such a three dimensional subspace as preserved by g. (25) is proved.

It remains only to observe that (23)-(24) imply that there exist proper invariant one and three dimensional subspaces of $g$ and that they are all degenerate. So $G$ is $S-W$ irreducible as claimed.

6. We conclude the proofs of the theorems announced in Section 1 by turning to Theorem 1 and Example 7. They are both straightforward.

Proof of Theorem 1. It is clear that the tangent space $M_{m}$ of $M$ at $m$ admits a decomposition into mutually orthogonal invariant subspaces of the holonomy group $H: M_{m}=M_{m}^{0} \oplus M_{m}^{1} \oplus \cdots \oplus M_{m}^{p}$, such that $H$ acts trivially on $M_{m}^{0}$, and acts weakly irreducibly on each $M_{m}^{i}, i=1, \cdots, p$. Parallel translation of these subspaces over $M$ gives rise to $(p+1)$ involutive distributions on $M$ which, by Proposition 3 of [15], induces a local isometry of $M$ at each point to a direct product which is compatible with the above decomposition of $M_{m}$. (Cf. 
Appendix II.) Hence the subgroup of $H$ generated by "small enough" closed paths is a direct product. To show that $H$ itself is actually a direct product of its normal subgroups $H^{i}, i=1, \cdots, p$, such that each $H^{i}$ acts weakly irreducibly on $M_{m}^{i}$ and acts trivially on all $M_{m}^{j}, j \neq i$, one must take care of arbitrary null homotopic paths. For this, one can employ the Factorization Lemma (p. 284-5 of [10]) in the same way as in the positive definite case. The details are given in p. 183-4 of [10], so we omit them.

Now if the maximal trivial subspace of $H$ in $M_{m}$ is nondegenerate, the above decomposition of $M_{m}$ is unique up to order. This last assertion is part of the Full de Rham Decomposition Theorem [15], a detailed statement as well as proof of which are given in Appendix I. It is then easy to see that the corresponding decomposition of $H$ is also unique up to order.

Proof of Assertions in Example 7. Let 0 be the origin of $\boldsymbol{R}^{3}$ and let $\left(u_{1}, u_{2}, u_{3}\right)$ be the canonical coordinates of $\boldsymbol{R}^{3}$. We adopt the convention that $E_{i}=\partial / \partial u_{i}$, and $e_{1}=\left(\partial / \partial u_{i}\right)(0), i=1,2,3$.

We new impose a riemannian structure on $\boldsymbol{R}^{3}$ from which the holonomy group will be computed directly. The metric and its inverse are:

$$
\begin{aligned}
g=\left\{g\left(E_{i}, E_{j}\right)\right\} & =\left[\begin{array}{ccc}
1+u_{2}^{4} & 0 & -u_{2}^{4} \\
0 & 1 & 0 \\
-u_{2}^{4} & 0 & -1+u_{2}^{4}
\end{array}\right] \\
g^{-1} & =\left[\begin{array}{ccc}
1-u_{2}^{4} & 0 & -u_{2}^{4} \\
0 & 1 & 0 \\
-u_{2}^{4} & 0 & -1-u_{2}^{4}
\end{array}\right]
\end{aligned}
$$

For the definitions and expressions in local coordinates of the Christoffel symbols $\Gamma_{j}^{i} k$, the curvature tensor $R_{E_{i} E_{j}}$ and its covariant derivatives $D_{E_{k}} R_{E_{i} E j}$, we refer once and for all to [10]. A computation then gives:

$$
\begin{array}{ll}
\Gamma_{11}^{1}=\Gamma_{11}^{3}=0 & \Gamma_{11}^{2}=-2 u_{2}^{3} \\
\Gamma_{12}^{1}=\Gamma_{12}^{3}=2 u_{2}^{3} & \Gamma_{12}^{2}=0 \\
\Gamma_{13}^{1}=\Gamma_{33}^{3}=0 & \Gamma_{13}^{2}=2 u_{2}^{3} \\
\Gamma_{22}^{1}=\Gamma_{22}^{3}=0 & \Gamma_{22}^{2}=0 \\
\Gamma_{23}^{1}=\Gamma_{23}^{3}=-2 u_{2}^{3} & \Gamma_{23}^{2}=0 \\
\Gamma_{33}^{1}=\Gamma_{33}^{3}=0 & \Gamma_{33}^{2}=-2 u_{2}^{3} .
\end{array}
$$

Since $\Gamma_{j 1}^{i}+\Gamma_{j 3}^{i} \equiv 0 \forall i, j$, we see that $D_{E_{j}}\left(E_{1}+E_{3}\right) \equiv 0, j=1,2,3$. Thus $E_{1}+E_{3}$ is an auto-parallel vector field on $\boldsymbol{R}^{3}$, which implies that $\mathfrak{h}$ must annihilate $\left(e_{1}+e_{3}\right)$. By a simple matrix computation, 


$$
\mathfrak{h} \leqq \operatorname{span}\{\alpha\}
$$

where

$$
\alpha=\left[\begin{array}{rrr}
0 & 1 & 0 \\
-1 & 0 & 1 \\
0 & 1 & 0
\end{array}\right]
$$

relative to $\left\{e_{i}\right\}$. Another computation shows that $R_{E_{1} E_{3}} \equiv 0$ but that $R_{E_{1} E_{2}}=R_{E_{2} E_{3}}=\left(-6 u_{2}^{2}\right) \alpha \neq 0$ relative to $\left\{E_{i}\right\}$. Ambrose-Singer's holonomy theorem implies immediately that $\mathfrak{h}$ is exactly span $\{\alpha\}$, and consequently, $H$ is the holonomy group of Theorem 3 (a).

Finally, $D_{E_{2}}\left(R_{E_{1} E_{2}}\right)=\left(12 u_{2}\right) \alpha \neq 0$. So, this riemannian manifold is not symmetric.

REMARK 7. This riemannian connection is unfortunately not complete. (It would have been complete if we had replaced $u_{2}^{4}$ by $u_{2}^{2}$ everywhere in the definition of $g$, in which case, the manifold is just the manifold of Theorem 3 (a)). For, let $\gamma:[0, a) \rightarrow \boldsymbol{R}^{3}$ be a geodesic and write $\gamma(t)=\left(u_{1}(t), u_{2}(t), u_{3}(t)\right)$. Then, $\gamma$ satisfies the differential equations of geodesics which in this case reduce to:

$$
\begin{aligned}
& u_{1}^{\prime \prime}+4 u_{2}^{3} u_{2}^{\prime}\left(u_{1}^{\prime}-u_{3}^{\prime}\right)=0 \\
& u_{2}^{\prime \prime}-2 u_{2}^{3}\left(u_{1}^{\prime}-u_{3}^{\prime}\right)^{2}=0 \\
& u_{1}^{\prime \prime}-u_{3}^{\prime \prime}=0
\end{aligned}
$$

where the primes denote differentiation with respect to $t$. Now if $u_{1}^{\prime}-u_{3}^{\prime}=k \neq 0$, the second equation becomes $u_{2}^{\prime \prime}=2 k^{2} u_{2}^{3}$ which contains a particular family of solutions of the form $(k t+b)^{-1}, b$ arbitrary. This family clearly has a singularity at $t=-b / k$, proving incompleteness. It would be interesting to have an example of a complete riemannian manifold which is both $S-W$ irreducible and nonsymmetric.

\section{Miscellaneous remarks.}

(A) Let $M$ be a simply connected symmetric space of dimension $d$ and let $I_{m}$ be the group of all isometries of $M$ leaving $m \in M$ fixed. If $f \in I_{m}$, then $(d f)_{m}$ is an isometry of $M_{m}$ onto itself such that if $(d f)_{m} \equiv g, g^{-1} \circ R_{g(x) g(y)} \circ g=R_{x y}$ for all $x, y \in M_{m}, R$ being the curvature tensor of $M$ at $m$. Furthermore $(d f)_{m}$ completely determines $f$. (This is a general fact true for an arbitrary affinely connected manifold. The usual proof for complete positive definite riemannian manifold does not carry over to the general case. Instead, one shows that if $f_{1}, f_{2}$ are two affine diffeomorphisms of $M$ onto itself such that $f_{1}(m)=f_{2}(m)$ and $\left(d f_{1}\right)_{m}=\left(d f_{2}\right)_{m}$ for some $m \in M$, then the set 


$$
\left\{n: n \in M, f_{1}(n)=f_{2}(n),\left(d f_{1}\right)_{n}=\left(d f_{2}\right)_{n}\right\}
$$

is both open and closed.) Thus $f \rightarrow(d f)_{m}$ is an isomorphism of $I_{m}$ onto a subgroup $\mathscr{F}_{m}$ of $P O\left(M_{m}\right) \subseteq G \mathrm{Y}\left(M_{m}\right)$. $\mathscr{F}_{m}$ is called the linear isotropy group of $M$ at $m$. We claim:

$$
\mathscr{F}_{m}=\left\{g \in P O\left(M_{m}\right): g^{-1} \circ R_{g(x) g(y)} \circ g=R_{x y}, \forall_{x}, y \in M_{m}\right\} .
$$

We have remarked already that $\mathscr{F}_{m}$ is contained in the right side. The reverse inclusion is an easy consequence of the Ambrose-Hicks Theorem [9] plus the that an affine diffeomorphism which induces an isometry between two tangent spaces is itself an isometry. Hence (26) is proved. Now, the set of curvture tensors on $M_{m}$ (see Definition 1) form a vector space $\mathscr{D}$, and if $g \in G Y\left(M_{m}\right)$, then $Q \rightarrow g Q$ such that $(g Q)_{x y}=g^{-1} \circ Q_{g(x) g(y)} \circ g$ defines an action of $G \mathfrak{r}\left(M_{m}\right)$ on $\mathscr{D}$. By (26), $\mathscr{F}_{m}$ is just the set of elements in $P O\left(M_{m}\right)$ leaving $R$ fixed under this action. In particular:

$$
\mathscr{F}_{m} \text { is a closed subgroup of } P O\left(M_{m}\right) \text {. }
$$

Now let $h$ be an element of the Lie algebra of $\mathscr{I}_{m}$. Since $(\exp h)$ $R=R$, one sees without difficulty that

$$
R_{h(x) y}+R_{x h(y)}+\left[R_{x y}, h\right]=0, \forall x, y \in M_{m} .
$$

In other words, (7) of Section 2 is satisfied. Hence,

(28) The Lie algebra of $\mathscr{J}_{m}$ is the set of elements of po $\left(M_{m}\right)$ which satisfy (7) of Section 2.

(B) On the basis of (A), we can compute the full isometry group $G$ of the symmetric spaces of Example 3. In general, given a symmetric space $M$, standard arguments show that the Lie algebra $g$ of $G$ is isomorphic to $\mathfrak{g}=\mathfrak{f} \oplus M_{m}$, where $\mathfrak{t}$ is the Lie algebra of $\mathscr{F}_{m}$, and the bracket operation is defined by (9) of Section 2. (Note that (28) insures that $g$ is in fact a Lie algebra.) Therefore, to compute $G$, it suffices to compute $g$, for which it in turn suffices to compute $\mathfrak{k}$. Here, we can avail ourselves of (28) and straightforward matrix calculations. We merely state the results.

For the space of Example 3 (a), $\mathfrak{i}=\mathfrak{l}$, the holonomy algebra. Therefore, as noted in the proof of Example 3 (a), the full group of isometries of this space is solvable.

For the space of Example $3(b), \mathfrak{l}$ is strictly bigger than the holonomy algebra; in fact $\mathfrak{f}$ coincides with the centralizer of $\mathfrak{h}$ in $\mathfrak{g} 0$ $(2,2)$. Precisely, relative to the same basis as in the statement of the theorem, let: 


$$
\begin{array}{rlrl}
\sigma_{1}=\left[\begin{array}{rrrr}
0 & 0 & 1 & 0 \\
0 & 0 & 0 & -1 \\
1 & 0 & 0 & 0 \\
0 & -1 & 0 & 0
\end{array}\right] & \sigma_{2}=\left[\begin{array}{rrrr}
0 & 1 & 0 & 0 \\
-1 & 0 & 0 & 0 \\
0 & 0 & 0 & 1 \\
0 & 0 & -1 & 0
\end{array}\right] \\
\sigma_{3}=\left[\begin{array}{rrrr}
0 & 0 & 0 & 1 \\
0 & 0 & 1 & 0 \\
0 & 1 & 0 & 0 \\
1 & 0 & 0 & 0
\end{array}\right] & \psi & \psi & =\left[\begin{array}{rrrr}
0 & -1 & 0 & 1 \\
1 & 0 & -1 & 0 \\
0 & -1 & 0 & 1 \\
1 & 0 & -1 & 0
\end{array}\right] .
\end{array}
$$

Then $\operatorname{span}\{\psi\}=\mathfrak{h}$ and $\mathfrak{t}=\operatorname{span}\left\{\dot{\psi}, \sigma_{1}, \sigma_{2}, \sigma_{3}\right\}$. Note that in this case, $g$ admits a nontrivial Levi decomposition:

$$
\operatorname{span}\left\{\sigma_{1}, \sigma_{2}, \sigma_{3}\right\} \oplus \operatorname{span}\left\{\psi, e_{1}, e_{2}, e_{3}, e_{4}\right\}
$$

where $\operatorname{span}\left\{\sigma_{1}, \sigma_{2}, \sigma_{3}\right\}$ is a Levi subalgebra.

(C) Still referring to the decomposition $\mathfrak{g}=\mathfrak{l} \oplus M_{m}$ of (B), since $a d\left(\left[M_{m}, M_{m}\right]\right)$ is the holonomy algebra $((8))$, and $a d(\mathfrak{f})$ is the linear isotropy algebra, it is clear that the holonomy algebra is an ideal in the linear isotropy algebra, i.e., $H$ is invariant in $\mathscr{J}_{m}$. Since $H$ can be solvable without being abelian, we see that $\mathscr{I}_{m}$ is not always reductive, in contrast with the positive definite case.

We note, in this connection, another anomaly of $\mathscr{I}_{m}$. It can happen that the linear isotropy group of a direct product of two weakly irreducible symmetric spaces is itself weakly irreducible. Hence, while the holonomy group of a symmetric space may be nondegenerately reducible, ([15] or [16]), the linear isotropy group can nonetheless be weakly irreducible. This explains why in the proof of Theorem 2, we chose to work with the subalgebra $a^{-}$, rather than with a itself.

To show this explicitly, let $M=\boldsymbol{R}^{4} \times \boldsymbol{R}^{4}$, where each factor is given the symmetric space structure of Theorem $3(b)$. Let

$$
\left\{e_{1}, \cdots, e_{4}\right\} \operatorname{span}\left(\boldsymbol{R}_{0}^{4}, 0\right) \text { and }\left\{f_{1}, \cdots, f_{4}\right\} \operatorname{span}\left(0, \boldsymbol{R}_{0}^{4}\right) \text {, }
$$

where $\boldsymbol{R}_{0}^{4}$ is the tangent space at the origin, and $\left\{e_{i}\right\}$ and $\left\{f_{i}\right\}$ are the kind of basis which occurred in the statement of the theorem. To avoid confusion, let $M_{0}^{1}=\operatorname{span}\left\{e_{1}, \cdots, e_{1}\right\}$ and $M_{0}^{2}=\operatorname{span}\left\{f_{1}, \cdots, f_{4}\right\}$, so that

$$
M_{0} \equiv \boldsymbol{R}_{0}^{4} \oplus \boldsymbol{R}_{0}^{4}=M_{0}^{1} \oplus M_{0}^{2} .
$$

We now make use of part (B). Let $\alpha$ (resp. $\beta)$ be the $8 \times 8$ matrix whose projection onto the upper left (resp. lower right) $4 \times 4$ bex be equal to $\psi$ (as in (B)) and equal to zero elsewhere. Let $\sigma_{1}, \sigma_{2}, \sigma_{3}$, (resp. $\sigma_{1}^{\prime}, \sigma_{2}^{\prime}, \sigma_{3}^{\prime}$ ) be the $8 \times 8$ matrices whose projections onto the upper 
left (resp. lower right) $4 \times 4$ box be equal to the $\sigma_{1}, \sigma_{2}, \sigma_{3}$ respectively of part (b), and equal to zero elsewhere. Further, let

$$
\begin{aligned}
& \gamma=\left[\begin{array}{rrrr|rrrr} 
& & & & 0 & -1 & 0 \\
& 0 & & & 0 & -1 \\
& & & & 0 & -1 & 0 \\
& & & 1 & 0 & -1 \\
\hline-1 & 0 & 1 & 0 & & & \\
0 & -1 & 0 & 1 & & & \\
-1 & 0 & 1 & 0 & & & & \\
0 & -1 & 0 & 1 & & &
\end{array}\right]
\end{aligned}
$$

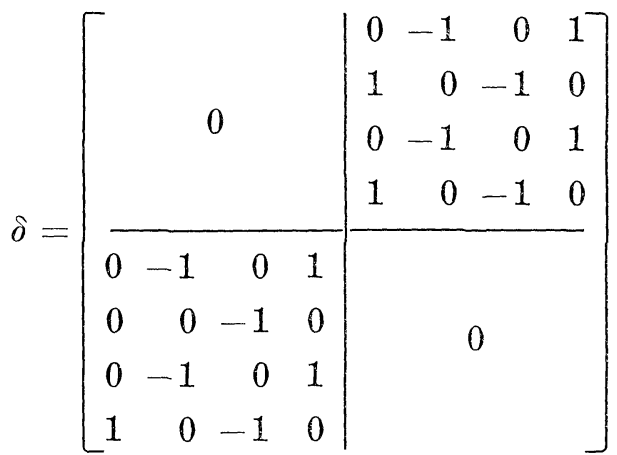

Then clearly, the holonomy algebra of $M$ equals $\operatorname{span}\{\alpha, \beta\}$, and one sees without difficulty that the linear isotropy algebra

$$
\mathfrak{k}=\operatorname{span}\left\{\alpha, \beta, \gamma, \delta, \sigma_{1}, \sigma_{2}, \sigma_{3}, \sigma_{1}^{\prime}, \sigma_{2}^{\prime}, \sigma_{3}^{\prime}\right\},
$$

the crucial fact here being:

$$
[\alpha, \gamma]=[\alpha, \delta]=[\beta, \gamma]=[\beta, \delta]=0 .
$$

We claim $\mathfrak{t}$ is weakly irreducible. In fact, one proves first that span $\left\{\alpha, \sigma_{1}, \sigma_{2}, \sigma_{3}\right\}$ has only one invariant subspace in $M_{0}^{1}$, namely, span $\left\{e_{1}+e_{3}, e_{2}+e_{\sharp}\right\}$. Similarly, the only invariant subspace of

$$
\operatorname{span}\left\{\beta, \sigma_{1}^{\prime}, \sigma_{2}^{\prime}, \sigma_{3}^{\prime}\right\}
$$

in $M_{0}^{2}$ is $\operatorname{span}\left\{f_{1}+f_{3}, f_{2}+f_{4}\right\}$. Now if $P$ is a subspace of $M_{0}$ left invariant by $\mathfrak{t}$, by considering the action of $\operatorname{span}\left\{\alpha, \sigma_{1}, \sigma_{2}, \sigma_{3}\right\}$ and $\operatorname{span}\left\{\beta, \sigma_{1}^{\prime}, \sigma_{2}^{\prime}, \sigma_{3}^{\prime}\right\}$ on $P$, one sees easily that $P$ is not of dimensions 1 or 3. If $P$ is two dimensional, then it is one of the above, and if it necessarily isotropic. Hence, $\mathfrak{l}$ is weakly irreducible, and so is $\mathscr{J}_{m}$.

(D) We retain the notation of (A). In analogy with the definitions of $I_{m}$ and $\mathscr{J}_{m}$, we let $I_{m}^{\prime}$ be the group of all affine diffeomor- 
phis four dimensional, then it is the direct sum of the above. So $P$ is phisms of $M$ onto itself leaving $m$ fixed, and let $\mathscr{F}_{m}^{\prime}$ be the isomorphic image of $I_{m}^{\prime}$ in $\operatorname{GI}\left(M_{m}\right)$ under $f \rightarrow(d f)_{m}$. Then again, the theorem of Ambrose. Hicks easily implies that

$$
\mathscr{F}_{m}^{\prime}=\left\{g \in G \mathbb{Y}\left(M_{m}\right): g^{-1} \circ R_{g(x) g(y)} \circ g=R_{x y}, \forall x, y \in M_{m}\right\} .
$$

The same reasoning as in $(\mathrm{A})$ shows that:

(29) $\mathscr{F}_{m}^{\prime}$ is a closed sudgroup of $G\left(M_{m}\right)$ and the Lie algebra of $\mathscr{F}_{m}^{\prime}$ is the set of elements of $\mathfrak{g l}\left(M_{m}\right)$ which satisfy (7) of Section 2.

According to Nomizu [13], when $M$ is positive definite riemannian and is irreducible, then $\mathscr{I}_{m}$ and $\mathscr{I}_{m}^{\prime}$ coincide. Passing on to the indefinite case and replacing, naturally, irreducibility by weak irreducibility, we see that this is no longer the case:

(30) The group of all affine diffeomorphisms of a weakly irreducible riemannian symmetric space onto itself is, in general, strictly bigger than the group of all isometries.

To show this, it suffices to verify in a particular case that

$$
\mathscr{F}_{m} \nsubseteq \mathscr{F}_{m}^{\prime} \text {. }
$$

We take the space of Example 3 (a). It was already remarked in (b) that the algebra of $\mathscr{I}_{m}$ is just $\operatorname{span}\{\alpha\}$, where

$$
\alpha=\left[\begin{array}{rrr}
0 & 1 & 0 \\
-1 & 0 & 1 \\
0 & 1 & 0
\end{array}\right],
$$

By a straightforward matrix computation, using (29), it can be seen that if

$$
\beta=\left[\begin{array}{lll}
2 & 0 & 0 \\
0 & 1 & 0 \\
2 & 0 & 0
\end{array}\right],
$$

then the algebra of $\mathscr{J}_{m}^{\prime}$ is $\operatorname{span}\{\alpha, \beta\}$. Hence the claim.

(E) Simons proved in [14], Theorem 8, that for an irreducible positive manifold of dimension $\geqq 3$, parallel translation of curvature being constant along closed paths is equivalent to its being constant along arbitrary paths. We see, from the proof of Theorem 7, that Simons' theorem is not true for weakly irreducible manifolds. For, if we denote the curvature tensor of that space at a point $p$ of $\boldsymbol{R}^{3}$ 
by $R$, then $\left\{\boldsymbol{R}_{p}^{3}, R, H\right\}$ is a symmetric holonomy system (see the proof of Theorem 3 (a)), and so by Remark 3 of Section 2, parallel translation of curvature is certainly constant along closed paths in that space. But that space is not symmetric.

(F) Finally, we would like to point out a curiosity. In the positive definite case, parallel translation preserving inner product implies that if a 1-dimensional subspace is left invariant by the holonomy group, then it is necessarily acted on trivially, i.e., every invariant 1-dimensional subspace is held fixed. While parallel translation still preserves inner product in the indefinite case, the isotropic vectors have an extra bit of freedom, and it is natural to ask if an invariant 1-dimensional isotropic subspace cannot be expanded and contracted by the holonomy group. The answer is yes: both for symmetric space and nonsymmetric spaces. For the symmetric case, an example is afforded by the space of Remark 6 of Section 5. There, both $\left(e_{3}-e_{5}\right)$ and $\left(e_{4}-e_{6}\right)$ are not annihilated by the holonomy algebra, but they both span invariant subspaces. For the nonsymmetric case, one can put a riemannian metric on $\boldsymbol{R}^{3}$ such as:

$$
g=\left[\begin{array}{ccc}
1+\left(u_{1}+u_{3}\right)^{2}+u_{2}^{2} & 0 & -\left(u_{1}+u_{3}\right)^{2}-u_{2}^{2} \\
0 & 1 & 0 \\
-\left(u_{1}+u_{3}\right)^{2}-u_{2}^{2} & 0 & -1+\left(u_{1}+u_{3}\right)^{2}+u_{2}^{2}
\end{array}\right]
$$

(We use the notation of the proof of Example 7.) It is a straightforward computation that $R_{E_{i} E_{j}}\left(E_{1}+E_{3}\right)=f_{i j}\left(E_{1}+E_{3}\right)$ where $f_{i j} \neq 0$ for all $i, j$. This shows that $\operatorname{span}\left\{e_{1}+e_{3}\right\}$ is invariant, but the action of $H$ on it is certainly nontrivial.

\section{APPENDIX I}

We stated in Section 5, (5) of [15] that the Full de Rham Decomposition Theorem (Section 5, (5)-A) can be proved in the same way as in the positive definite case. It has come to our attention that this is not entirely correct, and we now carefully restate it here-to bring the terminology up-to-date-and supply the missing link in the proof.

The Full De Rham Decomposition Theorem. Let $M$ be a complete, simply connected riemannian manifold and suppose the maximal trivial subspace $M_{m}^{o}$ of $H$ in $M_{m}$ is nondegenerate. ( $H$ denotes the holonomy group.) Then

(1) $M_{m}$ admits a decomposition into mutually orthogonal subspace which is unique up to order:

$$
M_{m}=M_{m}^{0} \oplus M_{m}^{1} \oplus \cdots \oplus M_{m}^{p}
$$


where each $M_{m}^{i}$ is left invariant by $H$, and $H$ acts weakly irreducibly on each $M_{m}^{i}, 1 \leqq i \leqq p$.

(2) $M$ is isometric to a direct product $M^{0} \times M^{1} \times \cdots \times M^{p}$ which is unique up to order, where $M^{j}, 0 \leqq j \leqq p$, is the maximal integral manifold of the distribution obtained by parallel translating $M_{m}^{j}$ over $M$. Moreover, $M^{0}$ is flat, and $M^{i}, 1 \leqq i \leqq p$ is weakly irreducible.

(3) $H$ is the direct prodct of itsu normal subgroups $H^{1} \times \cdots \times H^{p}$ which is unique up to order, where each $H^{i}$ is the holonomy group of $M^{i}, 1 \leqq i \leqq p$. Each $H^{i}$ is a weakly irreducible group and $H^{i}$ acts trivially on $M_{m}^{k}$, for $k \neq i$.

Proof. It is the uniqueness of $M_{m}^{0} \oplus M_{m}^{1} \oplus \cdots \oplus M_{m}^{p}$ that requires a new proof. All the rest then follows from this uniqueness fact.

Let $H=H^{1} \times \cdots \times H^{p}$ be the decomposition of $H$ associated with the above decomposition of $M_{m}$. Let $M_{m}=M_{m}^{0} \oplus N_{m}^{1} \oplus \cdots \oplus N_{m}^{r}$ be a second decomposition. For simplicity, let $N$ be any one of the $N_{m}^{\mathfrak{Y}}, 1 \leqq \mathfrak{l} \leqq r$. It suffices to prove that $N=M_{m}^{\alpha}$ for some $\alpha \in\{1, \cdots, p\}$.

Take $0 \neq n \in N$, and let $n=\left(n_{1}, \cdots, n_{p}\right)$ be the expression of $n$ relative to $M_{m}^{1} \oplus \cdots \oplus M_{m}^{p}$, i.e., $n_{i} \in M_{m}^{i}$ for all $i, 1 \leqq i \leqq p$. Let $n_{\alpha} \neq 0$ for some $\alpha$. Since the maximal trivial subspace $M_{m}^{0}$ has been split off, there exists a $g_{\alpha} \in H^{\alpha}$ such that $n_{\alpha} \neq g_{\alpha} n_{\alpha}$. Thus, since $H^{\alpha}$ acts trivially on $M_{m}^{k}$ if $k \neq \alpha$, we have:

$$
0 \neq\left(n_{\alpha}-g_{\alpha} n_{\alpha}\right)=\left(n-g_{\alpha} n\right) \in N \cap M_{m}^{\alpha} .
$$

We claim $N \cap M_{m}^{\alpha}$ is nondegenerate.

If not, there exists $v \in N \cap M_{m}^{\alpha}$ such that $\langle v, n\rangle=0$ for all $n \in N \cap M_{m}^{\alpha}$. Take an arbitrary $s \in N, s=\left(s_{1}, \cdots, s_{p}\right)$ such that

$$
s_{i} \in M_{m}^{i}, 1 \leqq i \leqq p,
$$

and take an arbitrary $g \in H, g=\left(g_{1}, \cdots, g_{p}\right)$ such that

$$
g_{i} \in H^{i}, 1 \leqq i \leqq p .
$$

As above, $\left(s-g_{\alpha} s\right) \in N \cap M_{m}^{\alpha}$. Thus $\left\langle v, s-g_{\alpha} s\right\rangle=0$, i.e.,

$$
\langle v, s\rangle=\left\langle v, g_{\alpha} s\right\rangle \text {. }
$$

Trivially, $\left\langle v, g_{\alpha} s\right\rangle=\langle v, g s\rangle$ because $g s=\left(g_{1} s_{1}, \cdots, g_{p} s_{p}\right)$ and the decomposition $M_{m}^{0} \oplus M_{m}^{1} \oplus \cdots \oplus M_{m}^{p}$ is orthogonal. So $\langle v, s\rangle=\langle v, g s\rangle$, or equivalently, $\langle g v, s\rangle=\langle v, s\rangle$ for all $s \in N, g \in H$. But the restriction of $\langle$,$\rangle to N$ is nondegenerate, hence $g v=r$ for all $g \in H$, which implies $v \in M_{m}^{0}$, contradiction.

So $N \cap M_{m}^{\alpha}$ is nondegenerate. Now $H$ acts weakly irreducibly on $M_{m}^{\alpha}$, so that of necessity $N \cap M_{m}^{\alpha}=M_{m}^{\alpha}$; otherwise, $N \cap M_{m}^{\alpha}$ will be a 
proper nondegenerate subspace of $M_{m}^{\alpha}$ left invariant by $H$. Reversing the roles of $N$ and $M_{m}^{\alpha}$, we have $N \cap M_{m}^{\alpha}=N$. Thus $N=M_{m}^{\alpha}$, and our claim is proved.

\section{APPENDIX II}

The statement of Theorem 3 of [16] is incorrect, but can be rectified simply as follows. Recall that a distribution $T$ is auto-parallel with respect to a connection iff the parallel translation of $T_{p}$ along any curve to another point $q \in M$ coincides with $T_{q}$. We say that a pair of supplementary auto-parallel distributions in an affinely-connected manifold $M$ induces a local affine decomposition of $M$ if and only if around each point $m \in M$, a neighborhood can be found which is affinely diffeomorphic to a neighborhood of $(m, m)$ of the direct product of the integral manifolds to the distributions through $m$. Similarly for local isometric decompositions. The correct version of Theorem 3 of [16] is then:

THEOREM. The following are equivalent for a riemannian $M$ :

(1) There exists a pair of supplementary auto-parallel distributions which induce a local affine decomposition of $M$.

(2) There exists a pair of supplementary auto-parallel distributions which induce a local isometric decomposition of $M$.

(3) The identity component of the holonomy group is nondegenerately reducible.

\section{REFERENCES}

1. W. Ambrose and I. M. Singer, A theorem on holonomy, Trans. Amer. Math. Soc. 75 (1953), 428-443.

2. M. Berger, Sur les groupes d'holonomie des variétés à connexion affine et des variétés riemanniennes, Bull. Soc. Math. France 83 (1953), 279-330.

3. - Les espaces symmétriques noncompacts, Ann. Ec. Norm. Sup. 74 (1957), 85-177.

4. A. Borel and A. Lichnerowicz, Groupes d'holonomie de variétés riemanniennes, C. R. Acad. Sc. Paris 234 (1952), 1835-1837.

5. N. Bourbaki, "Elements des mathématiques" Groupes et algebres de Lìe, Hermann, Paris, 1960.

6. C. Chevalley, On the topological structure of solvable groups, Ann. of Math. 42 (1941), 668-675.

7. M. Goto, Faithful representation of Lie groups I, Math. Japonica 1 (1948), 107-119.

8. S. Helgason, Differential geometry and Symmetric spaces, Academic Press, New York, 1962.

9. N. Hicks, A theorem on affine connexions, III. J. Math. 3 (1959), 242-254.

10. S. Kobayashi and K. Nomizu, Foundations of differential geometry, Interscience Publishers, New York, 1963.

11. A. Malcev, On the theory of Lie groups in the large, Matem. Sbornik, N. S. (58) 
16 (1945), 163-190.

12. A. Nijenhuis, On the holonomy group of linear connections, Indag. Math. 15 (1953), 233-249; 16 (1954), 17-25.

13. K. Nomizu, Invariant affne connections on homogeneous spaces, Amer. J. Math. 76 (1954), 33-65.

14. J. Simons, On transitivity of holonomy systems, Ann. of Math. 76 (1962), 213-234. 15. H. Wu, On the de Rham decomposition theorem, III. J. Math. 8 (1964), 291-311. 16. Decomposition of riemannian manifolds, Bull. Amer. Math. Soc. 70 (1964), 610-617.

Received April 27, 1965. Research supported by NSF GP-2439.

The Institute for Advanced Study PRINCETON, N. J. 


\section{PACIFIC JOURNAL OF MATHEMATICS}

\section{EDITORS}

\author{
H. SAMELSON \\ Stanford University \\ Stanford, California \\ J. P. JANS \\ University of Washington \\ Seattle, Washington 98105
}

\section{J. DugundJI}

University of Southern California Los Angeles, California 90007

RICHARD ARENS

University of California

Los Angeles, California 90024

\section{ASSOCIATE EDITORS}
E. F. BECKENBACH
B. H. NeumanN
F. WOLF
K. YOSIDA

\section{SUPPORTING INSTITUTIONS}

\author{
UNIVERSITY OF BRITISH COLUMBIA \\ CALIFORNIA INSTITUTE OF TECHNOLOGY \\ UNIVERSITY OF CALIFORNIA \\ MONTANA STATE UNIVERSITY \\ UNIVERSITY OF NEVADA \\ NEW MEXICO STATE UNIVERSITY \\ OREGON STATE UNIVERSITY \\ UNIVERSITY OF OREGON \\ OSAKA UNIVERSITY \\ UNIVERSITY OF SOUTHERN CALIFORNIA
}

\author{
STANFORD UNIVERSITY \\ UNIVERSITY OF TOKYO \\ UNIVERSITY OF UTAH \\ WASHINGTON STATE UNIVERSITY \\ UNIVERSITY OF WASHINGTON \\ AMERICAN MATHEMATICAL SOCIETY \\ CHEVRON RESEARCH CORPORATION \\ TRW SYSTEMS \\ NAVAL ORDNANCE TEST STATION
}

Mathematical papers intended for publication in the Pacific Journal of Mathematics should be typewritten (double spaced). The first paragraph or two must be capable of being used separately as a synopsis of the entire paper. It should not contain references to the bibliography. Manuscripts may be sent to any one of the four editors. All other communications to the editors should be addressed to the managing editor, Richard Arens at the University of California, Los Angeles, California 90024 .

50 reprints per author of each article are furnished free of charge; additional copies may be obtained at cost in multiples of 50 .

The Pacific Journal of Mathematics is published monthly. Effective with Volume 16 the price per volume (3 numbers) is $\$ 8.00$; single issues, $\$ 3.00$. Special price for current issues to individual faculty members of supporting institutions and to individual members of the American Mathematical Society: $\$ 4.00$ per volume; single issues $\$ 1.50$. Back numbers are available.

Subscriptions, orders for back numbers, and changes of address should be sent to Pacific Journal of Mathematics, 103 Highland Boulevard, Berkeley 8, California.

Printed at Kokusai Bunken Insatsusha (International Academic Printing Co., Ltd.), No. 6, 2-chome, Fujimi-cho, Chiyoda-ku, Tokyo, Japan.

PUBLISHED BY PACIFIC JOURNAL OF MATHEMATICS, A NON-PROFIT CORPORATION

The Supporting Institutions listed above contribute to the cost of publication of this Journal, but they are not owners or publishers and have no responsibility for its content or policies. 


\section{Pacific Journal of Mathematics \\ Vol. 20, No. $2 \quad$ October, 1967}

Edward Dewey Davis, Ideals of the principal class, $R$-sequences and a certain monoidal transformation ............................. 197

Richard Mansfield Dudley, Sub-stationary processes ................ 207

Newton Seymour Hawley and M. Schiffer, Riemann surfaces which are doubles of plane domains ......................... 217

Barry E. Johnson, Continuity of transformations which leave invariant certain translation invariant subspaces ................... 223

John Eldon Mack and Donald Glen Johnson, The Dedekind completion of

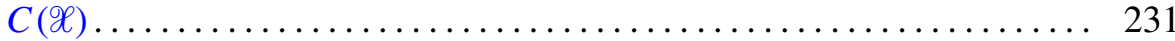

K. K. Mathur and R. B. Saxena, On the convergence of quasi-Hermite-Fejér interpolation................................... 245

H. D. Miller, Generalization of a theorem of Marcinkiewicz............ 261

Joseph Baruch Muskat, Reciprocity and Jacobi sums ............... 275

Stelios A. Negrepontis, On a theorem by Hoffman and Ramsay .......... 281

Paul Adrian Nickel, A note on principal functions and multiply-valent canonical mappings .............................. 283

Robert Charles Thompson, On a class of matrix equations ............. 289

David Morris Topping, Asymptoticity and semimodularity in projection lattices ........................................ 317

James Ramsey Webb, A Hellinger integral representation for bounded linear functionals

Joel John Westman, Locally trivial $C^{r}$ groupoids and their representations...

Hung-Hsi Wu, Holonomy groups of indefinite metrics 